\title{
Pluripotent Stem Cell Expansion and Neural Differentiation in 3-D Scaffolds of Tunable Poisson's Ratio
}

\author{
Yuanwei Yan $^{1}$, Yan $\mathrm{Li}^{2,3}$, Liqing Song ${ }^{1}$, Changchun Zeng ${ }^{2,3}$, Yan $\mathrm{Li}^{1, *}$
}

1. Department of Chemical and Biomedical Engineering, FAMU-FSU College of Engineering, Florida State University, Tallahassee, Florida, USA

2. Department of Industrial and Manufacturing Engineering, FAMU-FSU College of Engineering, Florida State University, Tallahassee, Florida, USA

3. The High-performance Materials Institute, Florida State University, Tallahassee, Florida, USA

Running title: Neural differentiation in 3-D auxetic scaffolds

* Corresponding author:

Dr. Yan Li: address: 2525 Pottsdamer St., Tallahassee, FL 32310, Tel: 850-410-6320; Fax: 850410-6150; email: yli@eng.fsu.edu

Submitted to Acta Biomaterialia 


\section{ABSTRACT:}

Biophysical properties of the scaffolds such as the elastic modulus, have been recently shown to impact stem cell lineage commitment. On the other hand, the contribution of the Poisson's ratio, another important biophysical property, to the stem cell fate decision, has not been studied. Scaffolds with tunable Poisson's ratio ( $v$ ) (termed as auxetic scaffolds when Poisson's ratio is zero or negative) are anticipated to provide a spectrum of unique biophysical 3D microenvironments to influence stem cell fate. To test this hypothesis, in the present work we fabricated auxetic polyurethane scaffolds $(v=0$ to -0.45$)$ and evaluated their effects on neural differentiation of mouse embryonic stem cells (ESCs) and human induced pluripotent stem cells (hiPSCs). Compared to the regular scaffolds $(v=+0.30)$ before auxetic conversion, the auxetic scaffolds supported smaller aggregate formation and higher expression of $\beta$-tubulin III upon neural differentiation. The influences of pore structure, Poisson's ratio, and elastic modulus on neural lineage commitment was further evaluated using a series of auxetic scaffolds. The results indicate that Poisson's ratio may confound the effects of elastic modulus, and auxetic scaffolds with proper pore structure and Poisson's ratio enhance neural differentiation. This study demonstrates that tuning the Poisson's ratio of the scaffolds together with elastic modulus and microstructure would enhance the capability to generate broader, more diversified ranges of biophysical 3-D microenvironments for the modulation of cellular differentiation.

Key words: Pluripotent stem cell, neural differentiation, auxetic scaffold, Poisson's ratio 


\section{Introduction}

Recently, generating neural cells and constructs from pluripotent stem cells (PSCs) has been shown as a promising platform to understand brain development for the applications in disease modeling and drug screening in order to treat neurological diseases [1-6]. During this process, biophysical signaling from the substrates/scaffolds plays a critical role in neural differentiation of PSCs [7-10]. For example, neural lineage commitment is very sensitive to the elastic modulus of the hydrogels or porous scaffolds, and low elastic modulus $(<10 \mathrm{kPa})$ promotes neuronal differentiation $[11,12]$. However, the understanding of these biophysical signals during PSC lineage commitment is very limited [8], and it is realized that the influence of different niche factors of substrates/scaffolds needs to be investigated [13]. The capability of a scaffolding material to transmit biophysical forces is mainly described by its elastic modulus $(E)$, and Poisson's ratio (v), the degree to which the materials contract or expand in the transverse direction compared to that of the longitudinal direction. Together, $E$ and $v$ define the materials deformation characteristics in three-dimensional (3-D) space, which influence PSC lineage commitment.

In addition to the materials with different $E$ that are used in most of previous studies [710], materials with the same $E$ but different $v$ will provide different 3 -D biophysical signals. For example, in a typical scaffold with a positive $v$ value, when the cells experience the tensile stress in the longitudinal direction, a compressive force would be exerted on the cells due to the contraction of the materials in the transverse direction. By contrast, a scaffold with a negative $v$ would expand in the transverse direction when tensile stress is applied in the longitudinal direction. This would result in the cells being stretched in all directions. Therefore, even if the two scaffolds have the same elastic modulus, the cell-matrix interactions would be substantially 
different if they have different Poisson's ratio. While the important influence of elastic modulus of biomaterials on stem cell differentiation has been revealed recently [7, 9, 12, 14-18], the impact of Poisson's ratio has barely been explored despite its importance. This is primarily due to 1) the scarcity of the materials with tunable Poisson's ratio as most existing materials (natural or man-made) have a narrow range of Poisson's ratio, typically $0.3-0.5$; and 2) lack of technology to produce materials with tailorable Poisson's ratio over a wide range.

Auxetic materials, the materials that display tunable Poisson's ratio in zero and negative range, have attracted great interests recently $[19,20]$, and recent studies have shown the important role of Poisson's ratio in several biophysical processes [21, 22]. For example, it has been reported that the Poisson's ratio of materials would affect cellular divisions [21, 23]. Moreover, embryonic stem cells (ESCs) exiting pluripotency were reported to exhibit auxetic nuclei shape $[24,25]$. Hence it might be desirable to use auxetic scaffolds to exert extracellular force and induce PSCs exiting pluripotency and modulate stem cell fate decisions [25]. In addition, materials with tunable Poisson's ratio would be more suitable to mimic the brain tissue environment than existing scaffold materials, as brain tissue has Poisson's ratio of nearly zero $[26,27]$ while most current materials in neural tissue engineering have Poisson's ratio of 0.3-0.5. In addition, PSC neural differentiation is mechanosensitive [28], and should be responsive to the biophysical microenvironment provided by auxetic materials.

Motivated by these advancements and challenges, the objective of this study is to evaluate the ability of the 3-D auxetic scaffolds with tunable biophysical properties ( $E$ and $v$ ) to influence PSC expansion and neural differentiation. Since auxetic conversion needs the modification of pore structure, the synergistic interactions of scaffold microstructure with $\mathrm{E}$ and $v$ were also studied. It is hypothesized that auxetic scaffolds with tunable $E$ and $v$ along with 
microstructure affect cell organization and transduce biophysical signals to modulate neural differentiation of PSCs. To test this hypothesis, auxetic scaffolds were fabricated and compared with the regular scaffolds that were used for the auxetic conversion in this study. Thereafter, a series of auxetic polyurethane scaffolds with different $v$ (0 to -0.45$), E$ (10 $\mathrm{kPa}$ to $100 \mathrm{kPa})$, and microstructure were fabricated and used to elucidate the contribution of each physical parameter to the cellular response. The influences of both regular scaffolds and auxetic scaffolds were evaluated on the cellular organization and neural differentiation of mouse ESCs and human induced pluripotent stem cells (hiPSCs). To our knowledge, this is the first study to investigate neural differentiation from PSCs in 3-D auxetic scaffolds.

\section{Materials and Methods}

\subsection{Undifferentiated PSC culture}

Murine ES-D3 line (American Type Culture Collection, Manassas, VA) was maintained on $0.1 \%$ gelatin-coated 6-well plates in a standard $5 \% \mathrm{CO}_{2}$ incubator as previously reported [29]. The expansion medium was composed of Dulbecco's Modified Eagle's Medium (DMEM, Life Technologies, Carlsbad, CA) supplemented with 10\% ESC-screened fetal bovine serum (FBS, Hyclone, Logan, UT), $1 \mathrm{mM}$ sodium pyruvate, $0.1 \mathrm{mM} \beta$-mercaptoethanol, penicillin (100 $\mathrm{U} / \mathrm{mL}$ ), streptomycin $(100 \mu \mathrm{g} / \mathrm{mL}$ ) (all from Life Technologies), and $1000 \mathrm{U} / \mathrm{mL}$ leukemia inhibitory factor (LIF, Millipore). The cells were seeded at $2-4 \times 10^{4}$ cells $/ \mathrm{cm}^{2}$ and sub-cultured with $0.05 \%$ trypsin/EDTA (Invitrogen) every $2-3$ days.

Human iPSK3 cells were derived from human foreskin fibroblasts transfected with plasmid DNA encoding reprogramming factors OCT4, NANOG, SOX2 and LIN28 (kindly provided by Dr. Stephen Duncan, Medical College of Wisconsin) [30, 31]. The human iPSK3 cells were maintained in mTeSR serum-free medium (StemCell Technologies, Inc., Vancouver, 
Canada) on 6-well plates coated with growth factor reduced Geltrex (Life Technologies). The cells were passaged by Accutase dissociation every 5-6 days and seeded at $1 \times 10^{6}$ cells per well of 6-well plate in the presence of $10 \mu \mathrm{M}$ Y27632 (Sigma) for the first 24 hours [32].

\subsection{Fabrication of auxetic scaffolds}

The auxetic scaffolds were fabricated by auxetic conversion of open cellular polyurethane foams (regular scaffolds) that showed compatibility in neutral differentiation [33], using technologies developed in our previous studies [34-36]. Briefly, the auxetic conversion is achieved by tri-axially compressing (compress in all three dimensions) the regular scaffolds under temperatures higher than a characteristic system transition temperature to partially buckle the foam cell ribs to generate re-entrant structures. Figure 1A (top) shows schematically the conversion of this structure and Figure 1B (top) illustrates the auxetic behavior such re-entrant structure would result. The regular scaffolds were referred as $\mathrm{R}$ and the auxetic scaffolds were referred as Au.

\subsection{Measurements of elastic modulus and Poisson's ratio}

Tensile tests were performed using a tensile tester (Shimadzu ASG-J) at a strain rate of 6 $\mathrm{mm} / \mathrm{min}$ and maximum strain of $10 \%$. The elastic modulus was determined by using the slope of the initial portion of the stress-strain curve, following standard methodology in mechanical property testing [34-36]. Determinations of Poisson's ratio (v) and elastic modulus $(E)$ of the scaffolds were performed based on video data acquired during tensile tests from a video extensometer system (Shimadzu DV-201) [34-36] . The video data were first transformed into static image data via the software MATLAB 2013b. Then, the length $(l)$ and diameter $(d)$ of the sample for every image were analyzed and determined using a MATLAB routine. Based on the length and the diameter, the transverse strain $\left(\varepsilon_{x}\right)$ and longitudinal strain $\left(\varepsilon_{y}\right)$ were calculated, 
respectively, using the following equations: $\varepsilon_{x}=\frac{\Delta l}{l_{0}}$ and $\varepsilon_{y}=\frac{\Delta d}{d_{0}}$, where, $l_{0}$ is the original length and $d_{0}$ is the original diameter. The Poisson's ratio was calculated following the definition of Poisson's ratio: $v=-\frac{\varepsilon_{x}}{\varepsilon_{y}}$.

\subsection{PSC seeding and cultivation on the scaffolds}

Prior to seeding, the scaffolds $(10 \mathrm{~mm} \times 10 \mathrm{~mm} \times 1 \mathrm{~mm})$ were washed with phosphate buffer saline (PBS) and sterilized with $70 \%$ ethanol. After extensive rinsing with PBS, the scaffolds were transferred into the wells of low attachment 24 -well plates. For ES-D3 cells, the scaffolds were coated with $0.1 \%$ gelatin for $30 \mathrm{~min}$. About $0.5 \times 10^{6}$ ES-D3 cells in $100 \mu \mathrm{L}$ of expansion medium were seeded into each scaffold. For human iPSK3 cells, the scaffolds were coated with $1 \%$ Geltrex for one hour. About $1.0 \times 10^{6}$ cells in mTeSR medium were seeded into each scaffold. After incubation overnight, the cell-seeded scaffolds were transferred into new wells with $1 \mathrm{~mL}$ of culture media. The seeding efficiency was about 30-35\%. The cells within scaffolds were cultured for 3-5 days and then characterized for cell organization, proliferation, and pluripotent marker expressions.

\subsection{Biochemical assays}

MTT assay: The cells in the scaffolds were incubated with $5 \mathrm{mg} / \mathrm{mL} 3-(4,5-$ Dimethylthiazol-2-yl)-2,5-diphenyltetrazolium bromide (MTT, Sigma) solution at day 1, 3, and 5. Afterwards, the formazan crystals were hydrolyzed with dimethyl sulfoxide (Sigma) and the absorbance was measured at $500 \mathrm{~nm}$ using a microplate reader (Biorad, Richmond, CA). Glucose/Lactate assay: The supernatants were analyzed for glucose and lactate concentration with a YSI 2700 Select $^{\mathrm{TM}}$ Bioanalyzer (Yellow Spring Instruments, Yellow Spring, OH). The lactate yield from glucose $(\mathrm{mol} / \mathrm{mol})$ was calculated based on the ratio of lactate production to 
glucose consumption. Alkaline phosphatase (ALP) assay: The cell-seeded scaffolds were digested with lysing buffer (PBS with 1\% Triton X-100, 0.5\% sodium deoxycholate, 0.1\% SDS, $0.1 \mathrm{mg} / \mathrm{mL}$ PMSF, $3 \%$ aprotinin). Cell lysate was then mixed with substrate and alkaline buffer. The mixture was incubated at $37^{\circ} \mathrm{C}$ for 15 min and the reaction was stopped using $0.5 \mathrm{~N} \mathrm{NaOH}$ solution. The absorbance was read at $415 \mathrm{~nm}$ on a microplate reader (Bio-Rad).

\subsection{DNA assay}

Cell numbers in the scaffolds were measured using DNA assay [37]. DNA standard was prepared by dissolving salmon testes DNA in TEX (10 mM Tris, $1 \mathrm{mM}$ EDTA, $0.1 \%$ Triton X100 at $\mathrm{pH}$ 8) and a standard curve was constructed for each assay. The cells in the scaffolds were lysed with $0.1 \mathrm{mg} / \mathrm{mL}$ proteinase K (Fisher Scientific, Pittsburgh, PA) at $50^{\circ} \mathrm{C}$ overnight. The lysates $(100 \mu \mathrm{L})$ were mixed with $100 \mu \mathrm{L}$ of Picogreen (Molecular Probes, Eugene, OR) in a 96-well plate. The plate was incubated for $5 \mathrm{~min}$ in the dark and then read on a fluorescent plate reader (FLX800, Bioinstrument Inc., Winooski, VT).

\subsection{Aggregate size distribution}

The images of cell aggregates in the scaffolds after staining with MTT dye (for cell visualization) were captured by a phase contrast microscopy. The captured images were converted to binary images using ImageJ software (http://rsb.info.nih.gov/ij) and analyzed to obtain the Feret's diameter of each aggregate and the aggregate size distribution [38]. For the cells treated with cytochalasin D (Sigma), $2 \mu \mathrm{M}$ cytochalasin D was added to the cell-scaffold constructs two days after seeding. The images with or without treatment were captured 24 hours later and analyzed for aggregate size distribution.

\subsection{Scanning electron microscopy (SEM)}

To assess scaffolds only, samples without cells were cut using a razor blade and the exposed surface was sputter-coated with a thin layer of gold before observation. For PSCs 
grown in the scaffolds, the samples were washed with PBS, fixed in $2.5 \%$ glutaraldehyde for 30 60 min, and dehydrated in graded ethanol solutions. The samples were dried by hexamethyldisilazane evaporation, mounted, and sputter-coated with gold. Observations were made using a JEOL 7401 SEM (FEI, Hillsboro, OR) under low-vacuum conditions.

\subsection{Neural differentiation of PSCs grown on the scaffolds}

For neural differentiation, ES-D3 cells were seeded into scaffolds in neural differentiation medium composed of DMEM-F12 plus 2\% B-27 serum-free supplement (Life Technologies) [39, 40]. At day 4, all-trans retinoic acid (RA) (Sigma-Aldrich, St. Louis, MI) was added at $1 \mu \mathrm{M}$ in the differentiation medium. After another four days of RA treatment, the cells were harvested with $0.05 \%$ trypsin/EDTA and replated onto Geltrex-coated 6-well plates. Extensive neural structures were observed after replating for 3-5 days. The neural marker expression was assessed by immunocytochemistry (after replating) and flow cytometry (upon harvesting). For human iPSK3 cells, neural differentiation was induced with $0.1 \mu \mathrm{M}$ LDN193189 (Sigma) and 10 $\mu \mathrm{M}$ SB431542 (Sigma) in DMEM-F12 plus 2\% B-27 for 7 days [41]. Then the cells were treated with $10 \mathrm{ng} / \mathrm{mL}$ fibroblast growth factor-2 (Life Technologies) and $2 \mu \mathrm{M}$ RA. At day 15, the cells were harvested to analyze neural marker expression.

\subsection{Immunocytochemistry}

The samples were fixed with $4 \%$ paraformaldehyde (PFA) and permeabilized with 0.20.5\% Triton X-100 for intracellular markers, followed by blocking and incubating with mouse or rabbit primary antibody against: Oct-4, Nanog, Nestin, PAX6, $\beta$-tubulin III, and E-cadherin (Supplementary Table S1). After washing, the cells were incubated with the corresponding secondary antibody: Alexa Fluor 488 goat anti-Mouse $\mathrm{IgG}_{1}$, Alexa Fluor® 488 or 594 goat anti-Rabbit IgG (Life Technologies). For surface markers SSEA-1 and NCAM, no permeabilization was performed. The secondary antibodies used were Alexa Fluor® 488 goat 
anti-Mouse IgM (for SSEA-1) or goat anti-mouse $\mathrm{IgG}_{1}$ (for NCAM). For F-actin staining, the cells were incubated with Alexa Fluor ${ }^{\circledR} 594$ Phalloidin (Life Technologies). The samples were stained with Hoechst 33342 or 4,6-diamidino-2-phenylindole (DAPI) and visualized using a fluorescent microscope (Olympus IX70, Melville, NY) or a confocal microscope (Leica TCS SP2 AOBS, Bannockburn, IL).

\subsection{Flow cytometry}

To quantify the levels of various marker expression, the cells were harvested by trypsinization and analyzed by flow cytometry [37]. Briefly, $1 \times 10^{6}$ cells per sample were fixed with 4\% PFA and washed with staining buffer (2\% FBS in PBS). The cells were permeabilized with $100 \%$ cold methanol for intracellular markers, blocked, and then incubated with primary antibodies against Oct-4, Nestin, PAX6, or $\beta$-tubulin III followed by the corresponding secondary antibody (Supplementary Table S1). For surface markers, the cells were blocked and then incubated with primary antibodies against SSEA-1 or NCAM followed by the Alexa Fluor® 488 goat anti-Mouse IgM (for SSEA-1) or $\operatorname{IgG}_{1}$ (for NCAM). The cells were acquired with BD FACSCanto ${ }^{\mathrm{TM}}$ II flow cytometer (Becton Dickinson) and analyzed against isotype controls using FlowJo software.

\subsection{Statistical analysis}

Each experiment was carried out at least three times. The representative results are expressed as [mean \pm mean absolute deviation (MD)] unless otherwise noted. Student's t-test was performed for the comparison of two groups. ANOVA followed by Fisher's LSD post hoc tests were performed for multiple group comparisons. A $p$-value <0.05 was considered statistically significant.

\section{Results}




\subsection{Fabrication and characterization of auxetic scaffolds}

Auxetic polyurethane (PU) scaffolds were fabricated using isotropic compression (triaxial compression) [34-36] to produce the re-entrant structure by partial bucking of the cell ribs (Figure 1A, top). The SEM micrographs in Figure 1A (bottom) revealed the prevalence of the re-entrant structure in the auxetic scaffold (Au, Figure 1A, bottom right) after the auxetic conversion using a regular scaffold (R, bottom left). Figure 1B (top) illustrates the mechanism by which the re-entrant microstructure would result in the auxetic behavior. It is conceivable that materials with this type of structure would expand in the transverse direction when being stretched. Figure 1B (bottom) shows the shape change of the auxetic scaffold under tensile test. Notable expansion in the transverse direction was evident, confirming the auxetic behavior of the Au scaffold. The mechanical properties of both scaffolds were measured following the methods described in the experimental section. The Au scaffold had a Poisson's ratio of -0.45 and elastic modulus of $44 \mathrm{kPa}$ (pore size range $250-300 \mu \mathrm{m}$, porosity $90.65 \pm 0.92 \%$ ), whereas the regular scaffold had a Poisson's ratio of 0.30 and elastic modulus of $100 \mathrm{kPa}$ (pore size range 300-400 $\mu \mathrm{m}, 96.31 \pm 0.16 \%)$. The regular scaffolds $(\mathrm{R})$ and the scaffolds after auxetic conversion $(\mathrm{Au})$ were then evaluated in the following study for PSC growth and neural differentiation.

\subsection{ESC growth, organization, and pluripotent marker expression in auxetic scaffolds}

Mouse ESCs seeded into auxetic $(\mathrm{Au})$ and regular $(\mathrm{R})$ scaffolds organized into cell aggregates associated with the scaffolds (Figure 2A and 2B). Noticeably, smaller aggregates were observed in the Au scaffolds compared to the R scaffolds. Cell organization inside auxetic scaffolds was also confirmed in a confocal image (Figure 2C), showing cellular aggregates associated with the porous structure of the scaffolds. The cells forming the aggregates displayed typical physiological morphology with microvilli as seen in SEM images (Figure 2D and 
Supplementary Figure S1). Average aggregate diameter was $76 \pm 22 \mu \mathrm{m}$ for the Au group and $145 \pm 48 \mu \mathrm{m}$ for the R group (Figure 2E). MTT activity showed cell viability and metabolic activity of the cells in the scaffolds (Figure 2F). Higher MTT activity was observed for the Au group compared to the $\mathrm{R}$ group. Cell expansion was observed over 3 days and from the cell number kinetics (Figure 2G), the doubling time was 23.5 hours for the cells grown in the $\mathrm{Au}$ scaffolds or 28.7 hours for cells grown in the R scaffolds. The lactate to glucose ratio reflects the metabolic activity of the cells. The cells in Au scaffolds had the ratio of $1.45 \pm 0.29(\mathrm{~mol} / \mathrm{mol})$, lower than the ratio for the $\mathrm{R}$ scaffolds at $1.68 \pm 0.30(\mathrm{~mol} / \mathrm{mol})$. The smaller aggregates in $\mathrm{Au}$ scaffolds may result in less diffusion limitation of the oxygen and nutrients compared to the larger aggregates in $\mathrm{R}$ scaffolds, leading to the lower lactate to glucose ratio.

Undifferentiated marker expression was assessed for the cells grown in different scaffolds. The cells harvested from both scaffolds resumed the morphology of undifferentiated colonies when replating onto gelatin-coated surface. The cells expressed high levels of undifferentiated markers SSEA-1, Oct-4, and Nanog for both groups (Figure 3A). Consistently, flow cytometry analysis showed $91.9 \%$ SSEA- $-1^{+}$and $91.0 \%$ Oct- $4^{+}$cells for the Au group, and 88.3\% SSEA- $1^{+}$and $87.1 \%$ Oct $-4^{+}$cells for the R group (Figure 3B). The in situ Oct-4 expression was examined by confocal microscopy and the aggregates in both scaffolds displayed a high level of Oct-4 expression (Figure 3C). ALP activity, another indicator of the undifferentiated cells, was comparable for the cells grown in the two scaffolds (Figure 3D).

\subsection{Neural differentiation of ESCs in auxetic scaffolds}

After 8 days of differentiation in the scaffolds, the ES-D3 cells were harvested and replated on Geltrex-coated surface for 5 days. Significant amounts of neurites/axons grew out of the small clumps and formed extensive neural networks (Figure 4A). Consistently, extensive 
expression of $\beta$-tubulin III, a marker for mature neurons, was observed for the cells from both scaffolds. The expression of neural cell adhesion molecule (NCAM), the marker for late neural progenitors, was also observed. By contrast, little expression of Nestin (a marker for early neural progenitor cells) was observed. Neural marker expression was further quantified by flow cytometry (Figure 4B). $\beta$-tubulin III expression was found significantly higher for the Au group compared to the R group ( $81 \pm 9 \%$ vs. $59 \pm 5 \%)$. The expression of NCAM was lower for the Au

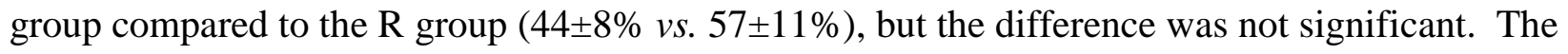
expression of Nestin was low and comparable for the cells from the two scaffolds, demonstrating neural lineage commitment toward more mature cells. Taken together, differential expression of neural markers was observed in the Au and the R scaffolds.

\subsection{Human iPSK3 cell growth and differentiation in auxetic scaffolds}

Neural differentiation from hiPSCs was performed in the Au scaffolds comparing with the R scaffolds (Figure 5). Consistently, the cells formed aggregates or clusters in the scaffolds (Figure 5A). At day 3, the average diameter of the aggregates was $90 \pm 38 \mu \mathrm{m}$ for the Au group and $124 \pm 42 \mu \mathrm{m}$ for the $\mathrm{R}$ scaffolds (Figure 5B). At day 5, the aggregate size increased to $126 \pm 47 \mu \mathrm{m}$ for the Au group and $186 \pm 57 \mu \mathrm{m}$ for the $\mathrm{R}$ group. Higher MTT activity was observed for the Au group compared to the R group (Figure 5C). In situ expression of Oct-4 and Nanog in the cells grown in both scaffolds were also observed (Figure 5D). The cells harvested from both scaffolds expressed a high level of pluripotent marker Nanog (Supplementary Figure S2). Upon neural differentiation, the cells from both scaffolds expressed neural progenitor markers Nestin and PAX6, along with a portion of more mature neural cells expressing $\beta$-tubulin III (Figure 6A). The cells from the Au scaffolds had 
significantly higher Nestin $(61.2 \pm 9.7 \%$ vs. $47.0 \pm 4.6 \%)$ and $\beta$-tubulin III $(55.1 \pm 1.6 \%$ vs. 46.5 $\pm 5.3 \%$ ) expression compared to the cells from the R scaffolds (Figure 6B, 6C).

\subsection{Effect of cytochalasin D treatment}

Because the major difference of cells grown in Au or R scaffolds was the aggregate size distribution, which may contribute to different metabolic activities and differential neural marker expression, the effect of cytochalasin $\mathrm{D}$, an actin depolymerization agent, on the cell organization in both scaffolds was examined. For Au scaffolds, the size of the aggregates was notably larger after cytochalasin D treatment (Figure 7A). The average diameter changed from $70 \pm 24 \mu \mathrm{m}$ to $111 \pm 37 \mu \mathrm{m}$ after the treatment. For R scaffolds, after the cytochalasin $\mathrm{D}$ treatment, there was little change (from $122 \pm 45 \mu \mathrm{m}$ to $111 \pm 32 \mu \mathrm{m}$ ) in the aggregate size distribution (Figure 7B). This observation might indicate the presence of aggregate compaction and contraction mediated by actin cytoskeleton for the cells grown in the auxetic scaffolds. F-actin distribution in the aggregates grown in both scaffolds was then examined for mouse ES-D3 cells and human iPSK3 cells (Figure 7C and Supplementary Figure S3). Dense F-actin expression inside the aggregates was observed for the Au group, while F-actin was uniformly distributed around cell-cell contacts throughout the aggregates for the $\mathrm{R}$ group. With cytochalasin D treatment, disorganized actin distribution was observed for both groups. These observations suggest that aggregate contraction in the Au scaffolds may be influenced by flexible re-entrant structure, in a different way from the cells in the $\mathrm{R}$ scaffolds with reticulate structure.

Additional studies were performed with Rho-associated kinase (ROCK) inhibitor Y27632 treatment for F-actin localization and E-cadherin expression (Supplementary Figure S4). Y27632 treatment resulted in more localized F-actin distribution. E-cadherin is an additional marker for cell organization/cell-matrix interactions [42]. Cells in auxetic scaffolds had more 
heterogeneous E-cadherin expression (some areas have higher intensity than other areas) while cells in regular scaffolds had more homogeneous expression. Y27632 treatment slightly increased E-cadherin expression. Further study is required for quantitative analysis of the role of the Rho/Rock/Myosin signaling axis in order to understand the cellular mechanism in cells grown in different scaffolds.

\subsection{Neural differentiation in scaffolds with different elastic modulus and Poisson's ratio}

To further ascertain the contribution of different biophysical properties of the auxetic PU scaffolds, a series of auxetic scaffolds (No. 2 to No. 6 in Table 1) was fabricated, and their morphology was shown in Figure 8A. For reference, the regular scaffold R was included as scaffold No. 1. These scaffolds can be divided into several groups to allow for de-convoluting the confounded influences of different biophysical properties. Scaffold No. 1-3 had similar elastic modulus $E(83-100 \mathrm{kPa})$ but different Poisson's ratio v (+0.30 vs. 0 vs. -0.45$)$. Scaffolds No. 4 and 5 had similar pore structure, similar $E(55-61 \mathrm{kPa})$, but different $v(0$ vs. -0.30$)$. Scaffold No. 6 had similar $v(-0.45)$ to scaffold No. 3, but different $E(10 \pm 1 \mathrm{kPa} v s .94 \pm 5 \mathrm{kPa})$.

Neural differentiation of ESCs on the six scaffolds was induced and neural marker expression (PAX6, Nestin, and $\beta$-tubulin III) for the cells grown in different scaffolds was quantified (Figure 8B and Supplementary Figure S5). The group of No. 3 scaffolds ( $E=94 \pm 5$ $\mathrm{kPa} ; \mathrm{v}=-0.45)$ had the highest expression of all three neural markers $\left(72.8 \pm 3.4 \% \mathrm{PAX6}^{+}\right.$, $62.4 \pm 3.6 \% \mathrm{Nestin}^{+}$, and $37.9 \pm 1.6 \% \beta$-tubulin $\mathrm{III}^{+}$), which was significantly higher than No. 1 $\left(E=100 \pm 5 \mathrm{kPa} ; \mathrm{v}=0.30 ; 39.1 \pm 2.3 \% \mathrm{PAX6}^{+}, 40.1 \pm 6.8 \% \mathrm{Nestin}^{+}, 27.2 \pm 4.9 \% \beta\right.$-tubulin $\left.\mathrm{III}^{+}\right)$, and No. $2\left(E=83 \pm 4 \mathrm{kPa} ; v=0.03 ; 49.0 \pm 9.8 \% \mathrm{PAX6}^{+}, 44.2 \pm 5.2 \% \mathrm{Nestin}^{+}\right.$, and $21.1 \pm 7.5 \% \beta$-tubulin

$\left.\mathrm{III}^{+}\right)($Figure 8Bi). $\quad \beta$-tubulin III expression for No. 4 scaffolds $(E=61 \pm 3 \mathrm{kPa} ; \mathrm{v}=0.01)$ was significantly higher $(32.8 \pm 5.8 \%$ vs. $18.4 \pm 3.0 \%)$ than No. 5 scaffolds $(E=55 \pm 3 \mathrm{kPa} ; \mathrm{v}=-0.30)$ 
(Figure 8Bii). The group of No. 3 scaffolds ( $E=94 \pm 5 \mathrm{kPa} ; \mathrm{v}=-0.45$ ) also expressed higher levels of neural markers than No. $6\left(E=10 \pm 1 \mathrm{kPa} ; \mathrm{v}=-0.45 ; 41.5 \pm 2.7 \% \mathrm{PAX}^{+}, 47.0 \pm 4.1 \% \mathrm{Nestin}^{+}\right.$, and $11.9 \pm 4.6 \% \beta$-tubulin $\mathrm{III}^{+}$) scaffolds (Figure 8Biii). Nestin expression for No. 3 scaffolds was significantly higher $(62.4 \pm 3.6 \%$ vs. $50.1 \pm 3.7 \%)$ than No. 4 scaffolds $(E=61 \pm 3 \mathrm{kPa} ; \mathrm{v}=0.01)$ (Figure 8Biv). The cells from all the scaffolds were harvested and replated on Geltrex-coated surface. Majority of the cells were neuronal with extensive neurite extension and $\beta$-tubulin III expression (Supplementary Figure S6 and S7). Overall, the results demonstrate differential expression of neural markers for the cells grown in the scaffolds with different biophysical properties. In particular, No.3 ( $E=94 \pm 5 \mathrm{kPa} ; \mathrm{v}=-0.45)$ and No.4 scaffolds $(E=61 \pm 3 \mathrm{kPa}$; $v=0.01)$ promoted neural marker expression compared to other scaffolds.

\section{Discussion}

Scaffolds having a tunable Poisson's ratio have shown advantages in some specific biomedical applications. For example, auextic materials have been used for bioprostheses and artery stents due to the enhanced compressive strength and shear stiffness [43, 44]. Auxetic materials are also the candidate scaffolds for cardiac tissue repair by cell injection because they can overcome biaxial squeezing of the contracting myocardium that is the cause of significant cell death during injection $[45,46]$. Recently, Poisson's ratio of biomaterials was found to have influence on cellular divisions and cell morphology [21, 23]. All these studies indicate that the 3-D scaffolds with tunable Poisson's ratio provide special biophysical microenvironment and have unique biomedical implications.

In particular, stem cells not only adhere to but also pull on the substrates, thereby responding to the deformation characteristics of the scaffolds in all three axes, which were collectively determined by the elastic modulus and the Poisson's ratio [23, 47]. The elastic 
modulus alone only represents the influence of the force balance on the cells along the principal loading axis (loading direction) $[13,48]$, and does not reflect the complete 3 -D influence of the scaffold. To date, only a few studies have investigated the effects of Poisson's ratio on the cellular fate using adult fibroblasts $[21,23,26]$. The effects of Poisson's ratio on neural tissue development and the influence of 3-D biophysical microenvironment on lineage commitment of PSCs have not been well understood. Such understandings would be highly valuable because lineage commitment and neural construct formation from PSCs can provide good model systems to study neurological diseases and pathological progression [3, 49].

Our results demonstrate that auxetic scaffolds did not impact pluripotent marker expression when the cells were maintained at undifferentiated state, but enhanced neural differentiation of PSCs when the cells were grown under neural induction condition. It is postulated that cellular organization and cell aggregate contraction are responsible for the observed effects of the auxetic scaffolds on neural differentiation, since the effect of cell organization/aggregates has been revealed to be an important parameter during PSC lineage commitment $[50,51]$. This postulation is supported by the different response of cellular aggregates (aggregates size distribution and F-actin expression) in auxetic scaffolds with cytochalasin D treatment compared to those in regular scaffolds. Aggregates in auxetic scaffolds appeared to be more compact, which was indicated by dense F-actin expression inside the aggregates and the change in aggregate size distribution after cytochalasin D treatment.

The auxetic scaffolds studied herein differ from the regular scaffolds in the following biophysical properties, which provide a valuable platform to probe the effects of each physical parameter on the cellular behavior: 1) pore structure (size and geometry); (2) Poisson's ratio; and (3) elastic modulus (Table 1). Our results indicate that pore structure in combination with 
Poisson's ratio could enhance neural differentiation by comparing neural marker expression for the No. 3 group $(E=94 \pm 5 \mathrm{kPa} ; \mathrm{v}=-0.45)$ to the No. $1(E=100 \pm 5 \mathrm{kPa} ; \mathrm{v}=0.30)$ and No. $2(E=83 \pm 4$ $\mathrm{kPa} ; \mathrm{v}=0.03$ ) groups, where No. 3 scaffolds (with highest neural marker expression) had the smaller pore and a $v$ value of -0.45 . Comparing scaffold No. $4(E=61 \pm 3 \mathrm{kPa} ; v=0.01)$ and No. 5 $(E=55 \pm 3 \mathrm{kPa} ; \mathrm{v}=-0.30)$, where the two scaffolds had similar pore structure and $E(55-60 \mathrm{kPa})$ but different $v$, higher $\beta$-tubulin III expression was observed for No. 4 scaffolds with $v$ close to 0 . Unexpectedly No. 6 scaffolds $(E=10 \pm 1 \mathrm{kPa})$ supported lower neural marker expression compared to No. 3 scaffolds ( $E=94 \pm 5 \mathrm{kPa}$ ), which had similar $v(-0.45)$ but different $E$. It was postulated that Poisson's ratio may confound the effect of elastic modulus during neural differentiation of PSCs. Within the tested range in the present study, the pore structure and Poisson's ratio are the likely two factors that contribute to the enhanced neural differentiation in the auxetic scaffolds.

The proof-of-concept study presented herein demonstrates the feasibility of using auxetic scaffolds to facilitate neural differentiation of PSCs, which has immediate applications in drug testing and toxicity study [6]. More detailed understanding of the impact of the biophysical properties and the micro/nano structure of 3-D auxetic scaffolds requires further explorations. In particular, applying mechanical stretching or compression during PSC differentiation may be of interest to biomaterials field. Auxetic conversion of 3-D scaffolds relies on ingenious materials design and engineering, and to-date was only realized with selected materials. With the advancement of the manufacturing technologies of the auxetic materials, the array of the scaffolds can be further expanded. Current cellular interactions with the scaffolds are on the cell cluster levels (aggregates of hundreds of microns). For example, technologies to fabricate auxetic scaffolds with pore size of 1-10 $\mu \mathrm{m}$ using biocompatible materials would be highly 
desired, as such materials would enable the investigation of cell-scaffold interaction on the cellular level. Nonetheless, the critical role of biophysical cues of extracellular microenvironment has just been discovered recently $[7,17]$ and this study adds a novel angle to understand the impact of biophysical microenvironment on stem cell fate decision.

\section{Conclusions}

In this study, auxetic scaffolds were evaluated for expansion and neural differentiation of mouse ESCs and hiPSCs. Different aggregate size distribution was observed between the regular and auxetic scaffolds, which may contribute to different cellular behaviors. When induced for neural differentiation, cells in auxetic scaffolds exhibited higher neuronal marker expression compared to the scaffolds before auxetic conversion. The microstructure and the Poisson's ratio of auxetic scaffolds likely contribute to the enhanced neural differentiation. It is noted that Poisson's ratio may also confound the effect of elastic modulus. This study provides the first step to decouple the effects of elastic modulus and Poisson's ratio to elucidate the influence of biophysical properties of 3-D microenvironment on stem cell fate decisions.

\section{Acknowledgment}

The authors would like to thank Ms. Ruth Didier in FSU Department of Biomedical Sciences for her help in flow cytometry analysis and confocal microscopy. This work is supported by FSU start up fund, FSU Research Foundation GAP award, FSU COFRS award, FSU Planning Grant, and partially National Science Foundation (grant No. 1342192).

\section{Disclosures}

No competing financial interests exist.

\section{References}

[1] D.X. Yu, M.C. Marchetto, F.H. Gage, Therapeutic translation of iPSCs for treating neurological disease, Cell Stem Cell, 12 (2013) 678-688. 
[2] S.J. Engle, D. Puppala, Integrating human pluripotent stem cells into drug development, Cell Stem Cell, 12 (2013) 669-677.

[3] M.A. Lancaster, M. Renner, C.A. Martin, D. Wenzel, L.S. Bicknell, M.E. Hurles, T. Homfray, J.M. Penninger, A.P. Jackson, J.A. Knoblich, Cerebral organoids model human brain development and microcephaly, Nature, 501 (2013) 373-379.

[4] S.T. Rashid, G.J. Alexander, Induced pluripotent stem cells: from Nobel Prizes to clinical applications, J Hepatol, 58 (2013) 625-629.

[5] T. Vazin, K.A. Ball, H. Lu, H. Park, Y. Ataeijannati, T. Head-Gordon, M.M. Poo, D.V. Schaffer, Efficient derivation of cortical glutamatergic neurons from human pluripotent stem cells: a model system to study neurotoxicity in Alzheimer's disease, Neurobiol Dis, 62 (2014) 62-72.

[6] M.P. Schwartz, Z. Hou, N.E. Propson, J. Zhang, C.J. Engstrom, V.S. Costa, P. Jiang, B.K. Nguyen, J.M. Bolin, W. Daly, Y. Wang, R. Stewart, C.D. Page, W.L. Murphy, J.A. Thomson, Human pluripotent stem cell-derived neural constructs for predicting neural toxicity, Proc Natl Acad Sci U S A, 112 (2015) 12516-12521.

[7] Y. Sun, K.M. Yong, L.G. Villa-Diaz, X. Zhang, W. Chen, R. Philson, S. Weng, H. Xu, P.H. Krebsbach, J. Fu, Hippo/YAP-mediated rigidity-dependent motor neuron differentiation of human pluripotent stem cells, Nat Mater, 13 (2014) 599-604.

[8] D.E. Discher, D.J. Mooney, P.W. Zandstra, Growth factors, matrices, and forces combine and control stem cells, Science, 324 (2009) 1673-1677.

[9] A.J. Keung, P. Asuri, S. Kumar, D.V. Schaffer, Soft microenvironments promote the early neurogenic differentiation but not self-renewal of human pluripotent stem cells, Integr Biol (Camb), 4 (2012) 1049-1058.

[10] A.J. Keung, S. Kumar, D.V. Schaffer, Presentation counts: microenvironmental regulation of stem cells by biophysical and material cues, Annu Rev Cell Dev Biol, 26 (2010) 533556.

[11] J. Zoldan, E.D. Karagiannis, C.Y. Lee, D.G. Anderson, R. Langer, S. Levenberg, The influence of scaffold elasticity on germ layer specification of human embryonic stem cells, Biomaterials, 32 (2011) 9612-9621.

[12] K. Saha, A.J. Keung, E.F. Irwin, Y. Li, L. Little, D.V. Schaffer, K.E. Healy, Substrate modulus directs neural stem cell behavior, Biophys J, 95 (2008) 4426-4438.

[13] L.H. Han, X. Tong, F. Yang, Photo-crosslinkable PEG-based microribbons for forming 3D macroporous scaffolds with decoupled niche properties, Adv Mater, 26 (2014) 1757-1762.

[14] A.J. Engler, S. Sen, H.L. Sweeney, D.E. Discher, Matrix elasticity directs stem cell lineage specification, Cell, 126 (2006) 677-689.

[15] S. Musah, P.J. Wrighton, Y. Zaltsman, X. Zhong, S. Zorn, M.B. Parlato, C. Hsiao, S.P. Palecek, Q. Chang, W.L. Murphy, L.L. Kiessling, Substratum-induced differentiation of human pluripotent stem cells reveals the coactivator YAP is a potent regulator of neuronal specification, Proc Natl Acad Sci U S A, 111 (2014) 13805-13810. 
[16] A.J. Keung, M. Dong, D.V. Schaffer, S. Kumar, Pan-neuronal maturation but not neuronal subtype differentiation of adult neural stem cells is mechanosensitive, Sci Rep, 3 (2013) 1817.

[17] C. Yang, M.W. Tibbitt, L. Basta, K.S. Anseth, Mechanical memory and dosing influence stem cell fate, Nat Mater, 13 (2014) 645-652.

[18] S. Sart, Y. Yan, Y. Li, E. Lochner, C. Zeng, T. Ma, Y. Li, Crosslinking of extracellular matrix scaffolds derived from pluripotent stem cell aggregates modulates neural differentiation, Acta Biomater, 30 (2016) 222-232.

[19] K.E. Evans, A. Alderson, Auxetic Materials: Functional Materials and Structures from Lateral Thinking!, Adv Mater, 12 (2000) 617-628.

[20] G.N. Greaves, A.L. Greer, R.S. Lakes, T. Rouxel, Poisson's ratio and modern materials, Nat Mater, 10 (2011) 823-837.

[21] W. Zhang, P. Soman, K. Meggs, X. Qu, S. Chen, Turning the Poisson's ratio of biomaterials for investigating cellular response, Adv Funct Mater, 23 (2013) 3226-3232.

[22] R. Critchley, I. Corni, J.A. Wharton, F.C. Walsh, R.J.K. Wood, K.R. Stokes, A review of the manufacture, mechanical properties and potential applications of auxetic foams, Physica Status Solidi B, 250 (2013) 1963-1982.

[23] P. Soman, J.W. Lee, A. Phadke, S. Varghese, S. Chen, Spatial tuning of negative and positive Poisson's ratio in a multi-layer scaffold, Acta Biomater, 8 (2012) 2587-2594.

[24] S. Pagliara, K. Franze, C.R. McClain, G.W. Wylde, C.L. Fisher, R.J. Franklin, A.J. Kabla, U.F. Keyser, K.J. Chalut, Auxetic nuclei in embryonic stem cells exiting pluripotency, Nat Mater, 13 (2014) 638-644.

[25] N. Wang, Stem cell mechanics: Auxetic nuclei, Nat Mater, 13 (2014) 540-542.

[26] P. Soman, D.Y. Fozdar, J.W. Lee, A. Phadke, S. Varghese, S. Chen, A three-dimensional polymer scaffolding material exhibiting a zero Poisson's ratio, Soft Matter, 8 (2012) 4946-4951.

[27] S.K. Kyriacou, A. Mohamed, K. Miller, S. Neff, Brain mechanics For neurosurgery: modeling issues, Biomech Model Mechanobiol, 1 (2002) 151-164.

[28] Y. Shao, J. Sang, J. Fu, On human pluripotent stem cell control: The rise of 3D bioengineering and mechanobiology, Biomaterials, 52 (2015) 26-43.

[29] Y. Yan, F. Calixto Bejarano, S. Sart, M. Muroski, G.F. Strouse, S.C. Grant, Y. Li, Cryopreservation of embryonic stem cell-derived multicellular neural aggregates labeled with micron-sized particles of iron oxide for magnetic resonance imaging, Biotechnol Prog, 31 (2015) 510-521.

[30] K. Si-Tayeb, F.K. Noto, A. Sepac, F. Sedlic, Z.J. Bosnjak, J.W. Lough, S.A. Duncan, Generation of human induced pluripotent stem cells by simple transient transfection of plasmid DNA encoding reprogramming factors, BMC Dev Biol, 10 (2010) 81.

[31] K. Si-Tayeb, F.K. Noto, M. Nagaoka, J. Li, M.A. Battle, C. Duris, P.E. North, S. Dalton, S.A. Duncan, Highly efficient generation of human hepatocyte-like cells from induced pluripotent stem cells, Hepatology, 51 (2010) 297-305. 
[32] Y. Yan, L. Martin, D. Bosco, J. Bundy, R. Nowakowski, Q.X. Sang, Y. Li, Differential effects of acellular embryonic matrices on pluripotent stem cell expansion and neural differentiation., Biomaterials, 73 (2015) 231-242.

[33] B. Carlberg, M.Z. Axell, U. Nannmark, J. Liu, H.G. Kuhn, Electrospun polyurethane scaffolds for proliferation and neuronal differentiation of human embryonic stem cells, Biomed Mater, 4 (2009) 045004.

[34] C. Zeng, Y. Li, Materials systems and methods of manufacture for auxetic foams., US Patent Application, WO2016/014782A1 (2014).

[35] Y. Li, C. Zeng, On the successful fabrication of auxetic polyurethane foams: Materials requirement, processing strategy and conversion mechanism, Polymer, 87 (2016) 98-107.

[36] Y. Li, C. Zeng, Room-temperature, near-instantaneous fabrication of auxetic materials with constant Poisson's ratio over large deformation, Adv Mater, 28 (2016) 2822-2826.

[37] S. Sart, T. Ma, Y. Li, Extracellular matrices decellularized from embryonic stem cells maintained their structure and signaling specificity, Tissue Eng Part A 20 (2014) 54-66.

[38] S. Sart, T. Ma, Y. Li, Cryopreservation of pluripotent stem cell aggregates in defined protein-free formulation, Biotechnol Prog, 29 (2013) 143-153.

[39] S. Sart, Y. Liu, T. Ma, Y. Li, Microenvironment regulation of pluripotent stem cell-derived neural progenitor aggregates by human mesenchymal stem cell secretome, Tissue Eng Part A, 20 (2014) 2666-2679.

[40] S. Sart, Y. Yan, Y. Li, The microenvironment of embryoid bodies modulated the commitment to neural lineage post-cryopreservation, Tissue Eng Part C: Methods., 21 (2015) 356-366.

[41] Y. Yan, J. Bejoy, J. Xia, J. Guan, Y. Zhou, Y. Li, Neural patterning of human induced pluripotent stem cells in 3-D cultures for studying biomolecule-directed differential cellular responses, Acta Biomater, 42 (2016) 114-126.

[42] Y. Xu, X. Zhu, H.S. Hahm, W. Wei, E. Hao, A. Hayek, S. Ding, Revealing a core signaling regulatory mechanism for pluripotent stem cell survival and self-renewal by small molecules, Proc Natl Acad Sci U S A, 107 (2010) 8129-8134.

[43] F. Scarpa, Auxetic materials for bioprostheses [In the Spotlight] IEEE Signal Processing Magazine, 25 (2008) 126-128.

[44] B.D. Caddock, K.E. Evans, Negative Poisson ratios and strain-dependent mechanical properties in arterial prostheses, Biomaterials, 16 (1995) 1109-1115.

[45] C.J. Teng, J. Luo, R.C. Chiu, D. Shum-Tim, Massive mechanical loss of microspheres with direct intramyocardial injection in the beating heart: implications for cellular cardiomyoplasty, J Thorac Cardiovasc Surg, 132 (2006) 628-632.

[46] H. Jawad, A.R. Lyon, S.E. Harding, N.N. Ali, A.R. Boccaccini, Myocardial tissue engineering, Br Med Bull, 87 (2008) 31-47.

[47] D.Y. Fozdar, P. Soman, J.W. Lee, L.H. Han, S. Chen, Three-dimensional polymer constructs exhibiting a tunable negative poisson's ratio, Adv Funct Mater, 21 (2011) 2712-2720. 
[48] Y. Sun, C.S. Chen, J. Fu, Forcing stem cells to behave: a biophysical perspective of the cellular microenvironment, Annu Rev Biophys, 41 (2012) 519-542.

[49] J. Mariani, M.V. Simonini, D. Palejev, L. Tomasini, G. Coppola, A.M. Szekely, T.L. Horvath, F.M. Vaccarino, Modeling human cortical development in vitro using induced pluripotent stem cells, Proc Natl Acad Sci U S A, 109 (2012) 12770-12775.

[50] C.L. Bauwens, R. Peerani, S. Niebruegge, K.A. Woodhouse, E. Kumacheva, M. Husain, P.W. Zandstra, Control of human embryonic stem cell colony and aggregate size heterogeneity influences differentiation trajectories, STEM CELLS, 26 (2008) 2300-2310.

[51] J.C. Mohr, J. Zhang, S.M. Azarin, A.G. Soerens, J.J. de Pablo, J.A. Thomson, G.E. Lyons, S.P. Palecek, T.J. Kamp, The microwell control of embryoid body size in order to regulate cardiac differentiation of human embryonic stem cells, Biomaterials, 31 (2010) 1885-1893. 
Table 1. Summary of biophysical properties of different PU scaffolds.

\begin{tabular}{|c|c|c|c|c|c|}
\hline Scaffold ID & $\begin{array}{l}\text { Elastic } \\
\text { Modulus } \\
(\mathrm{kPa})\end{array}$ & $\begin{array}{l}\text { Poisson's } \\
\text { Ratio }\end{array}$ & Pore Geometry & $\begin{array}{l}\text { Pore Size } \\
(\mu \mathrm{m})^{*}\end{array}$ & Porosity \\
\hline No. 1 & $100 \pm 5$ & 0.30 & $\begin{array}{l}\text { Pentagonal } \\
\text { dodecahedron }\end{array}$ & $300-400$ & $96.31 \pm 0.16 \%$ \\
\hline No. 2 & $83 \pm 4$ & 0.03 & Re-entrant structure & $250-300$ & $94.73 \pm 0.27 \%$ \\
\hline No. 3 & $94 \pm 5$ & -0.45 & Re-entrant structure & $200-250$ & $85.55 \pm 0.35 \%$ \\
\hline No. 4 & $61 \pm 3$ & 0.01 & Re-entrant structure & $250-300$ & $94.99 \pm 0.38 \%$ \\
\hline No. 5 & $55 \pm 3$ & -0.30 & Re-entrant structure & $250-300$ & $94.58 \pm 0.41 \%$ \\
\hline No. 6 & $10 \pm 1$ & -0.45 & Re-entrant structure & $200-250$ & $88.98 \pm 1.32 \%$ \\
\hline
\end{tabular}




\section{Figure legends}

Figure 1. Fabrication and characterization of auxetic polyurethane scaffolds. (A) Top: schematics of the auxetic conversion process by partial buckling of the cell ribs using isotropic (tri-axial) compression; Bottom: Scanning electron microscopy (SEM) micrographs of the regular scaffold (left) and the auxetic scaffold (right). Scale bar: $500 \mu \mathrm{m}$. Note the extensive reentrant structures in the auxetic scaffold; (B) Top: a schematic illustration of the auxetic response from the re-entrant microstructure. When materials with the re-entrant microstructure are stretched, the inverse trapezoid would flatten, resulting in the expansion in the lateral direction; Bottom: photographs of the fabricated auxetic foam in the original (left) and stretched state (right). Note the considerable expansion in the lateral direction, which confirms the excellent auxetic property.

Figure 2. Cell organization, proliferation, and metabolic activities of mouse ESCs in auxetic scaffolds. (A) Phase contrast image of cell organization in auxetic scaffolds; white arrows: aggregates on the pore hinge; red arrow: aggregates in the pore; (B) phase contrast image of cell organization in regular scaffolds; scale bar: $200 \mu \mathrm{m} . \quad$ (C) Fluorescent image of cell organization in auxetic scaffolds; Blue-DAPI for cell nuclei showing aggregate structure; Redscaffolds; scale bar: $100 \mu \mathrm{m}$. (D) Scanning electron microscopy (SEM) image of cells grown in auxetic scaffolds (5000X), scale bar: $3 \mu \mathrm{m}$. (E) Aggregate size distribution (day 3) (n=107); (F) MTT activity of cells grown in different scaffolds; (G) Cell number in different scaffolds determined by DNA assay; $(\mathrm{H})$ Lactate to glucose ratio for cells grown in different scaffolds. * $p<0.05$. 
Figure 3. Undifferentiated marker expression of mouse ESCs in auxetic scaffolds. (A) Fluorescent images of SSEA-1, Oct-4 and Nanog expression of cells from different scaffolds (day 5); scale bar: $200 \mu \mathrm{m}$ for SSEA-1 and Oct4, $100 \mu \mathrm{m}$ for Nanog. (B) SSEA-1 and Oct-4 expression quantified by flow cytometry; (C) Confocal images of in situ Oct-4 expression of cells grown in different scaffolds; scale bar: $100 \mu \mathrm{m}$. Green: Oct-4; Blue: Hoechst 33342. Scaffolds exhibit blue fluorescence. (D) Alkaline phosphatase (ALP) expression.

Figure 4. Neural differentiation of mouse ESCs in auxetic scaffolds. (A) Fluorescent images of neural marker expression of replated cells from different scaffolds; scale bar: $200 \mu \mathrm{m}$. Green: neural marker of interest; Blue: Hoechst 33342. (B) Neural marker expression quantified by flow cytometry. (a) $\beta$-tubulin III; (b) NCAM; (c) Nestin. (i) Representative histograms from flow cytometry; (ii) quantification of neural marker expression $(\mathrm{n}=3) . * p<0.05$.

Figure 5. Cell organization and pluripotent marker expression of human iPSK3 cells grown in auxetic scaffolds. (A) Phase contrast images of cell organization in different scaffolds; white arrows: cell aggregates; red arrows: cell clusters; (B) Aggregate size distribution at (i) day 3 (n=115); (ii) day $5(\mathrm{n}=115)$. (C) MTT activity of cells grown in different scaffolds (from one representative experiment); (D) Confocal images of in situ Oct-4 and Nanog expression of cells grown in different scaffolds. Scale bar: $25 \mu \mathrm{m}$ for Oct-4 (Au and R) and Au Nanog. Scale bar: $100 \mu \mathrm{m}$ for R Nanog. * $p<0.05$.

Figure 6. Neural differentiation of human iPSK3 cells grown in auxetic scaffolds. (A) Fluorescent images of neural marker expression (Nestin and PAX6, Nestin and $\beta$-tubulin III) of replated cells from different scaffolds; scale bar: $100 \mu \mathrm{m}$. (B) Neural marker (Nestin and $\beta$ - 
tubulin III) expression after 15-day differentiation quantified by flow cytometry $(\mathrm{n}=3) . * p<0.05$.

(C) Representative histograms from flow cytometry.

Figure 7. Effect of cytochalasin D treatment on cell organization in auxetic scaffolds. (A) Phase contrast images of mouse ESC aggregates in different scaffolds with or without cytochalasin D treatment. The images were taken after staining with MTT dye. Scale bar: 200 $\mu \mathrm{m}$. (B) Aggregate size distribution of mouse ESC aggregates for different scaffolds with or without cytochalasin D treatment. (C) Confocal images of F-actin organization for human iPSK3 cell aggregates in different scaffolds with or without cytochalasin D treatment. Red: Factin; Blue: Hoechst 33342. Scale bar; $50 \mu \mathrm{m}$, (i) auxetic scaffolds (Au); (ii) regular scaffolds (R).

Figure 8. Neural differentiation of mouse ESCs grown in auxetic scaffolds with different elastic modulus and Poisson's ratio. (A) The series of auxetic scaffolds with different elastic modulus and Poisson's ratio (v). The images of different scaffolds (No.1 to No. 6) were captured using scanning electron microscopy (50X). Scale bar: $200 \mu \mathrm{m}$. The range of elastic modulus is $10 \mathrm{kPa}$ to $100 \mathrm{kPa}$. The range of Poisson's ratio is 0.3 to -0.45 . (B) Neural marker (Nestin, PAX6, and $\beta$-tubulin III) expression at day 6 differentiation quantified by flow cytometry $(\mathrm{n}=3)$. (i) compares scaffold \#1, \#2, \#3, which have similar elastic modulus, but different Poisson's ratio and pore size; (ii) compares scaffold \#4, \#5, which have similar elastic modulus and pore size, but different Poisson's ratio; (iii) compares scaffold \#3, \#6, which have similar Poisson's ratio and pore size, but different elastic modulus; (iv) compares scaffold \#3, \#4, the two best conditions which have different combinations of Poisson's ratio, elastic modulus, and pore size. $* p<0.05$. 


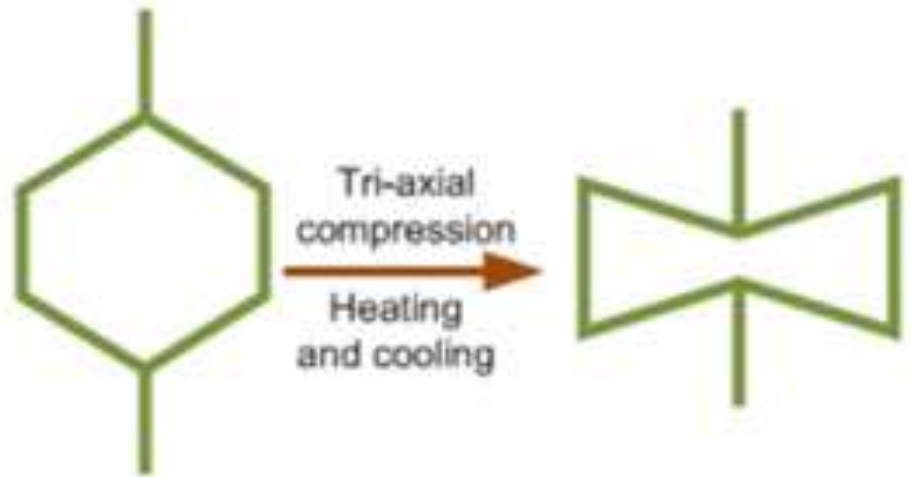

Regular (R)

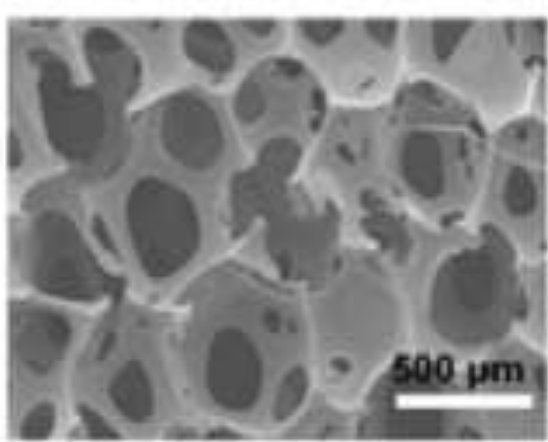

Auxetic (Au)

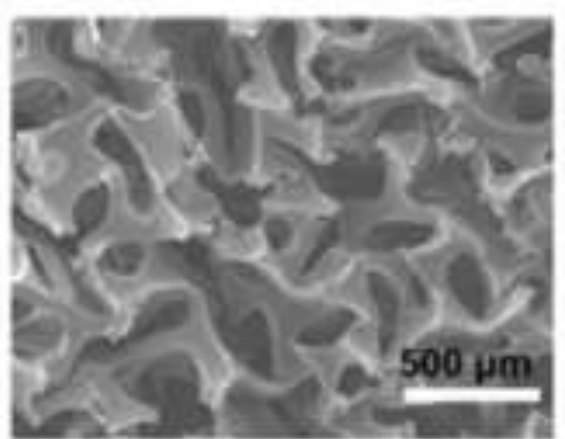

A

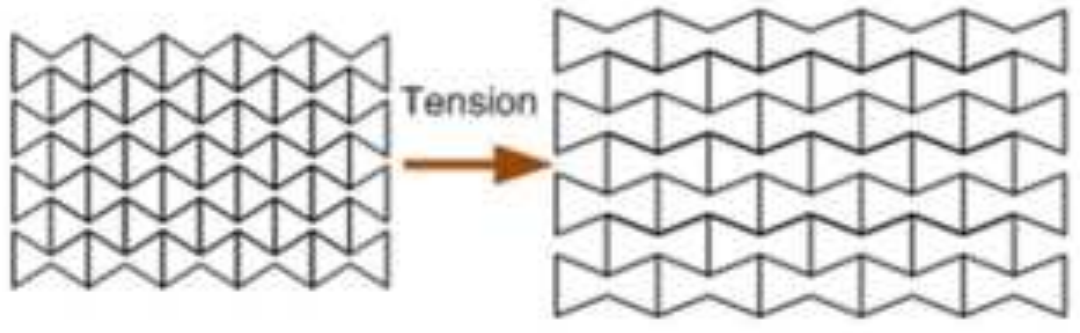

Original state
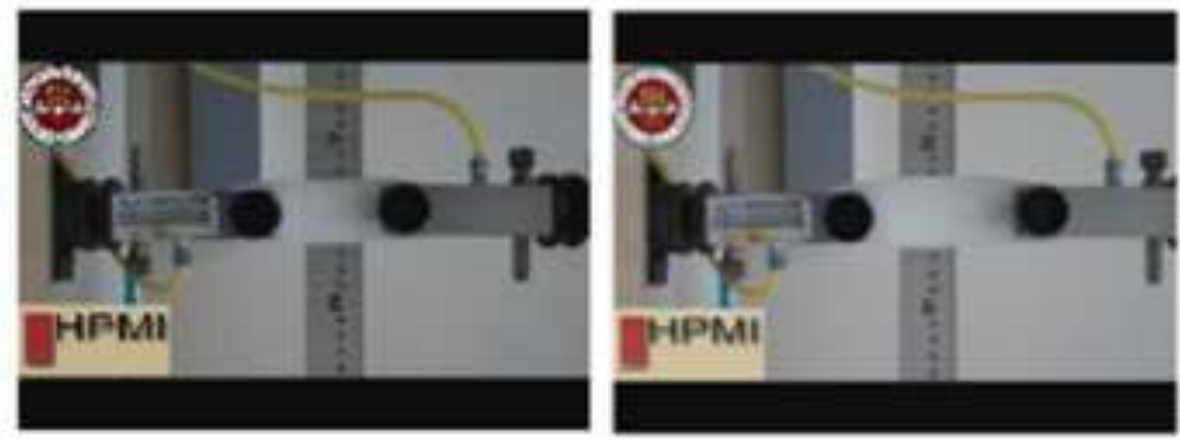

B 

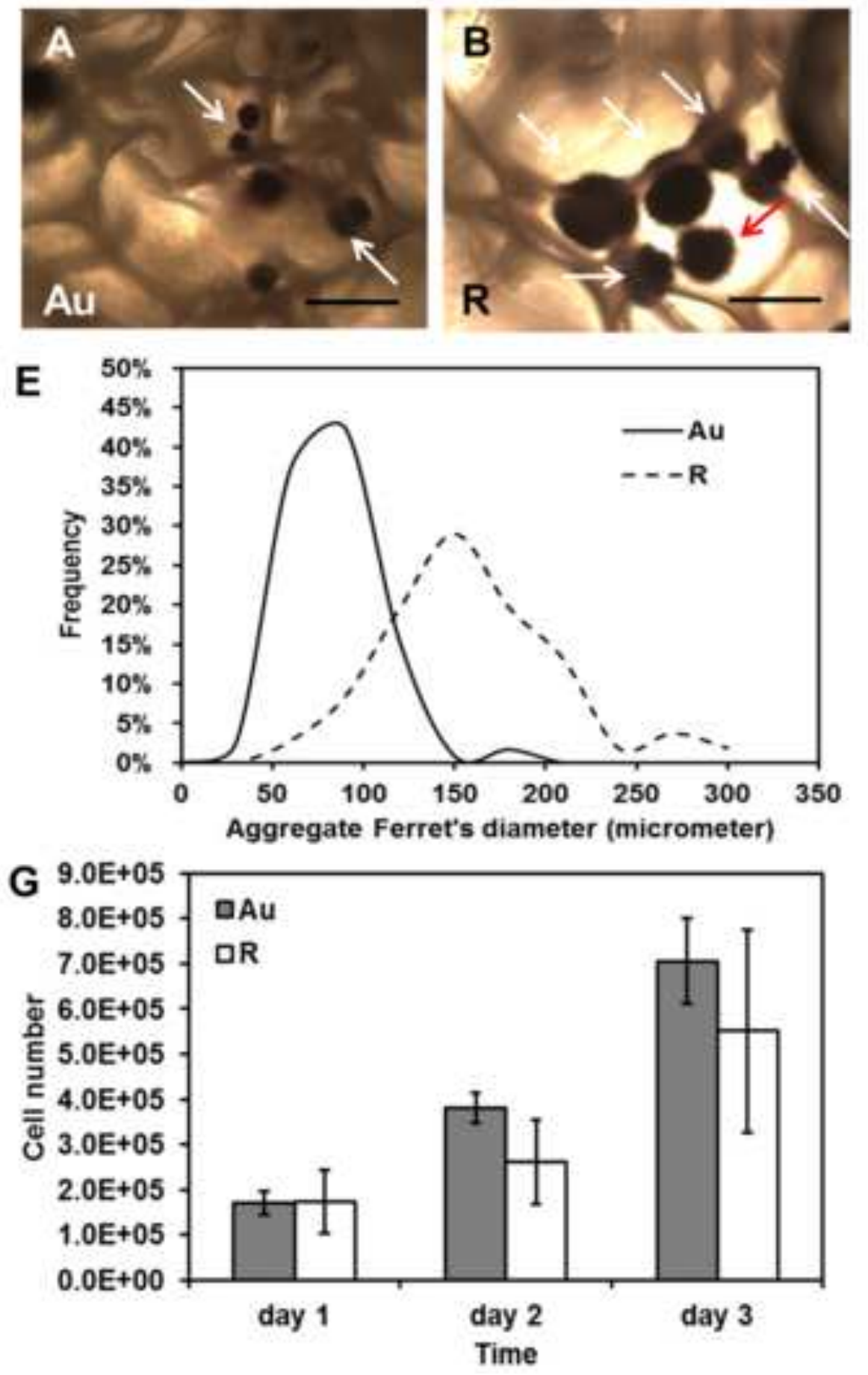
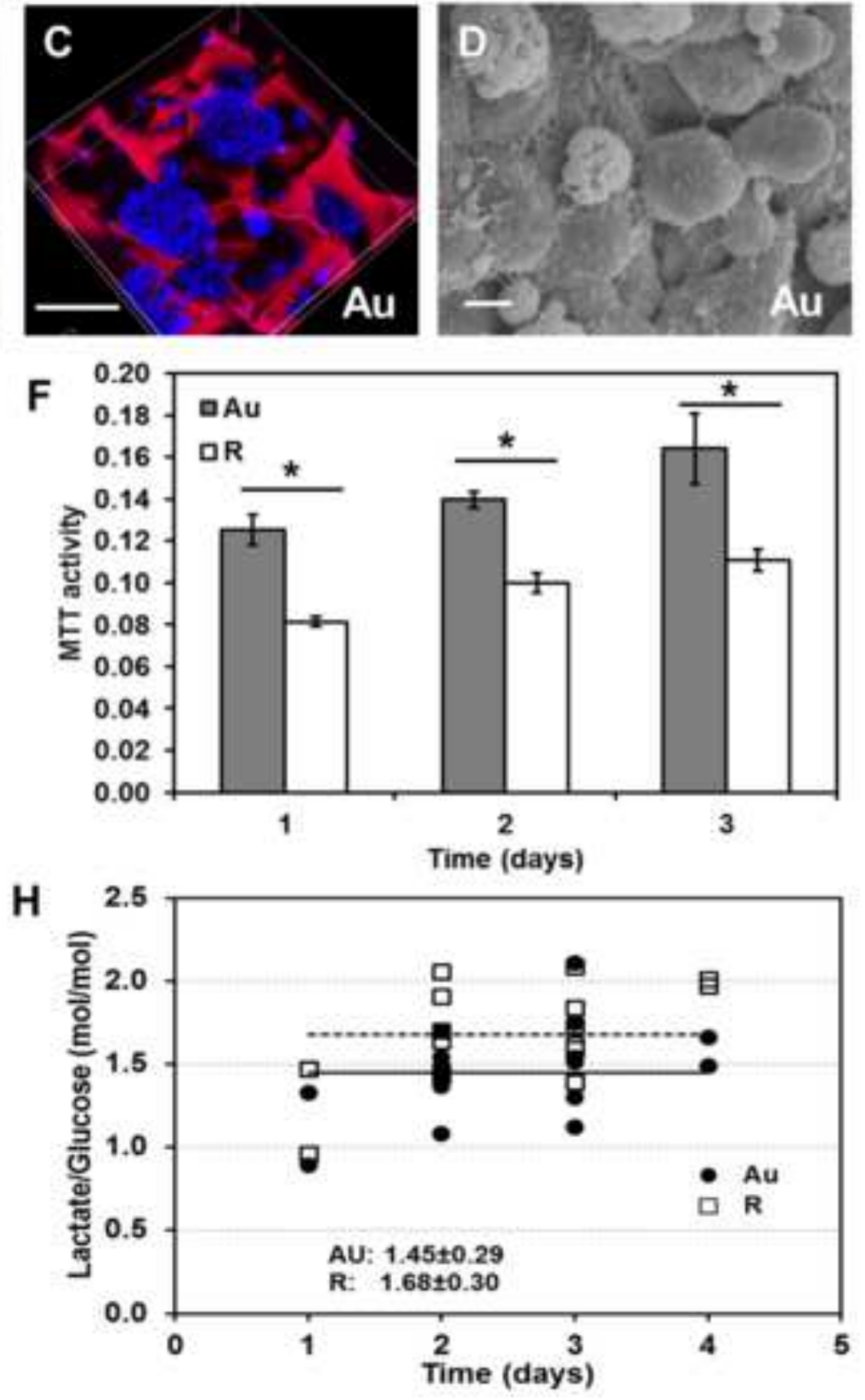

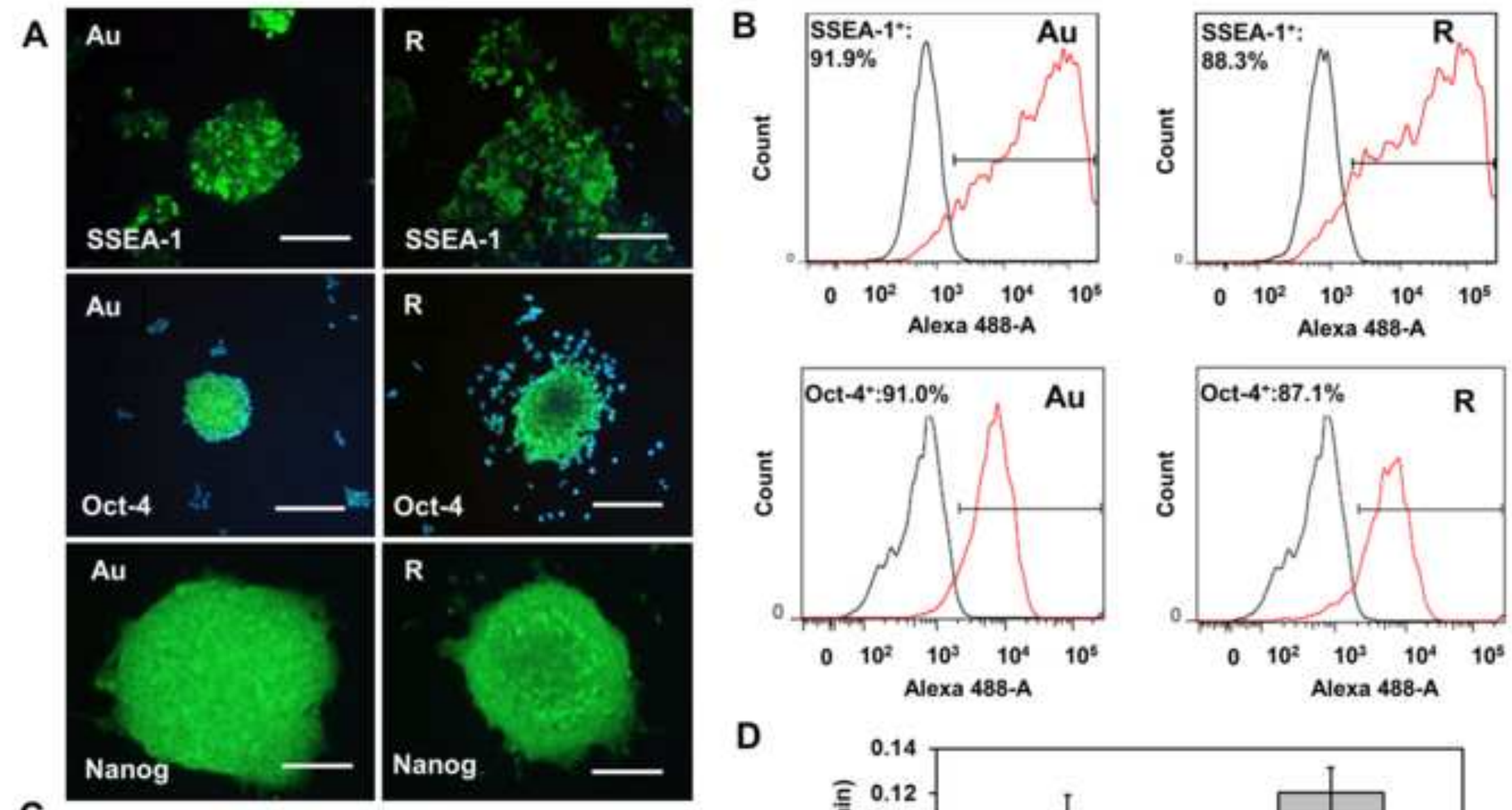

C

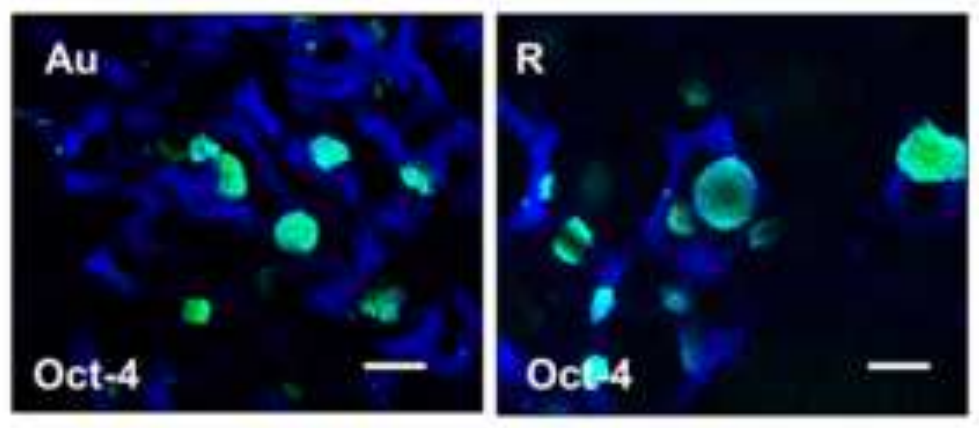

D

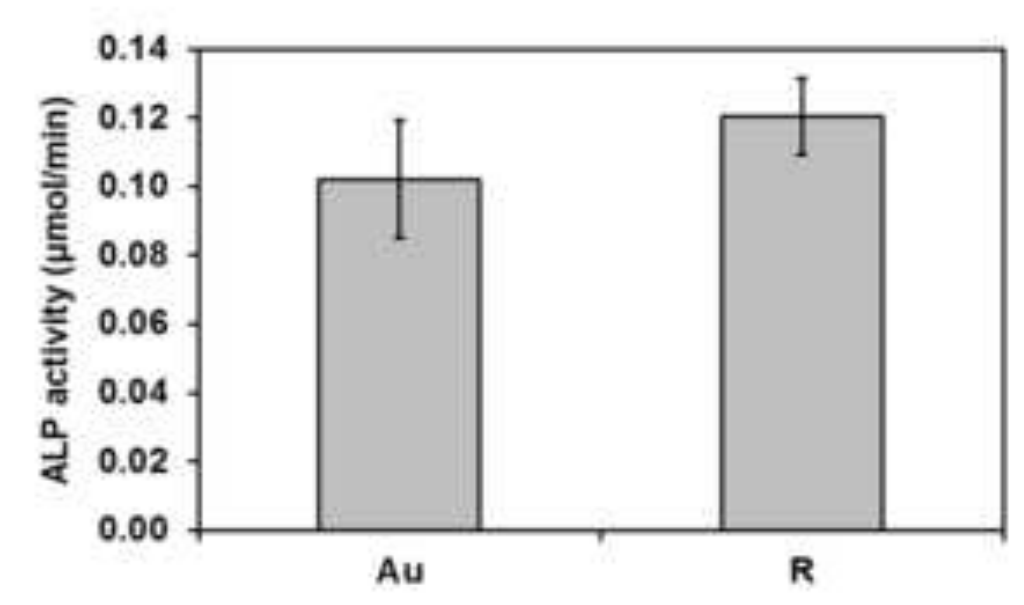


A
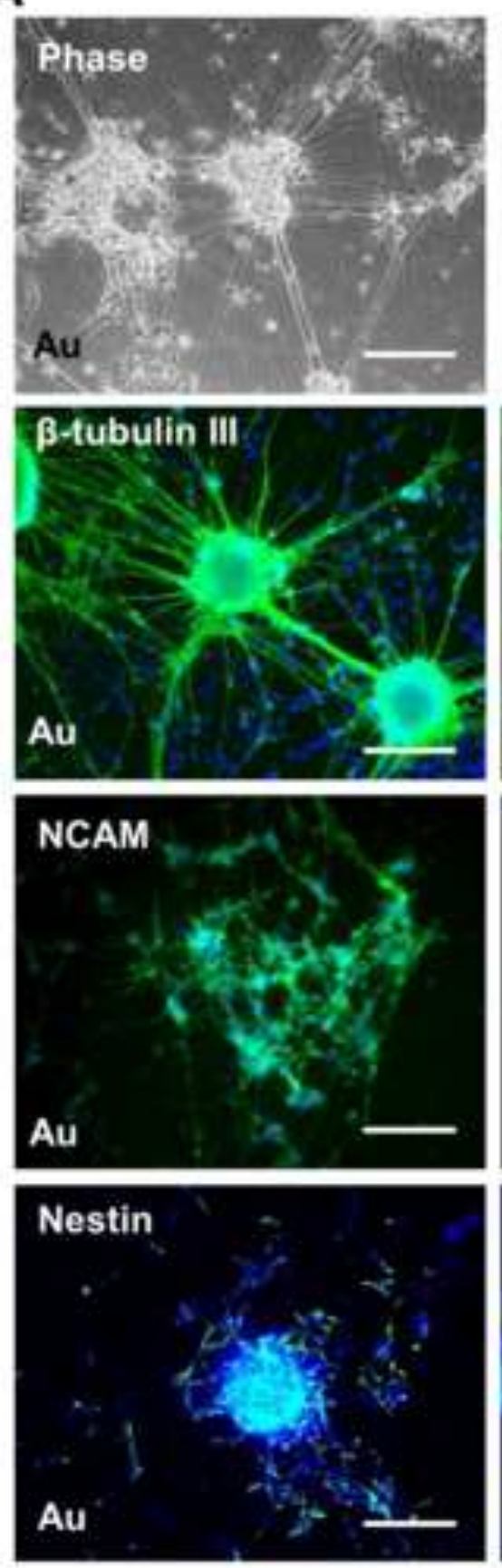
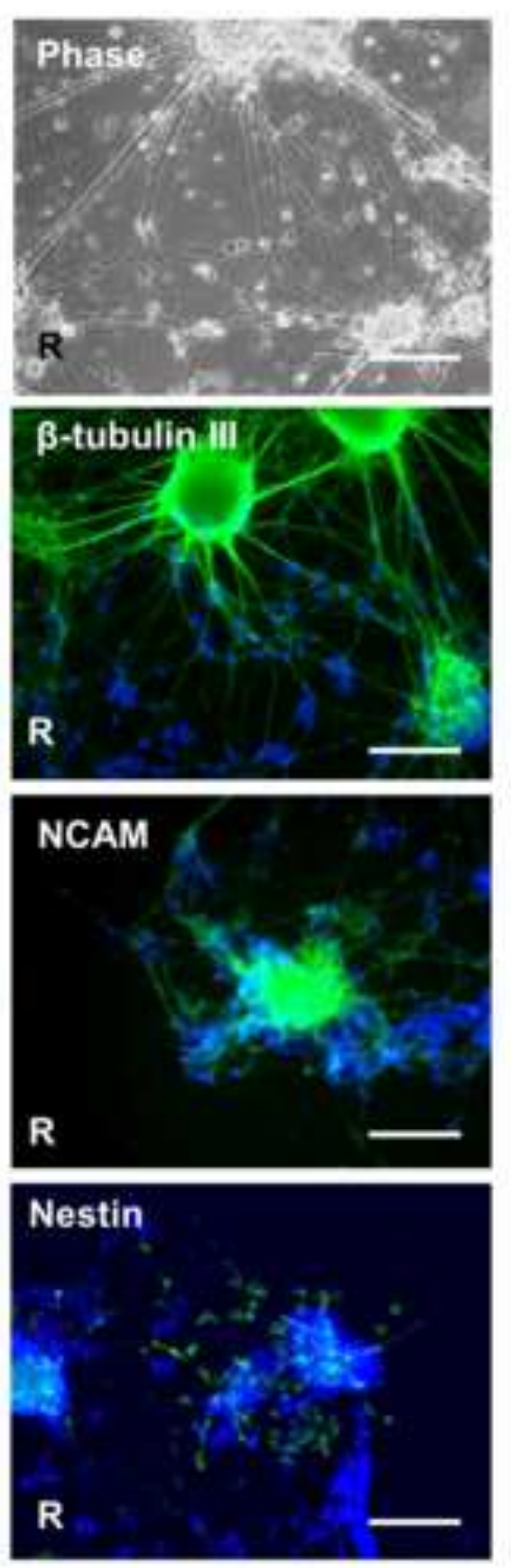

B
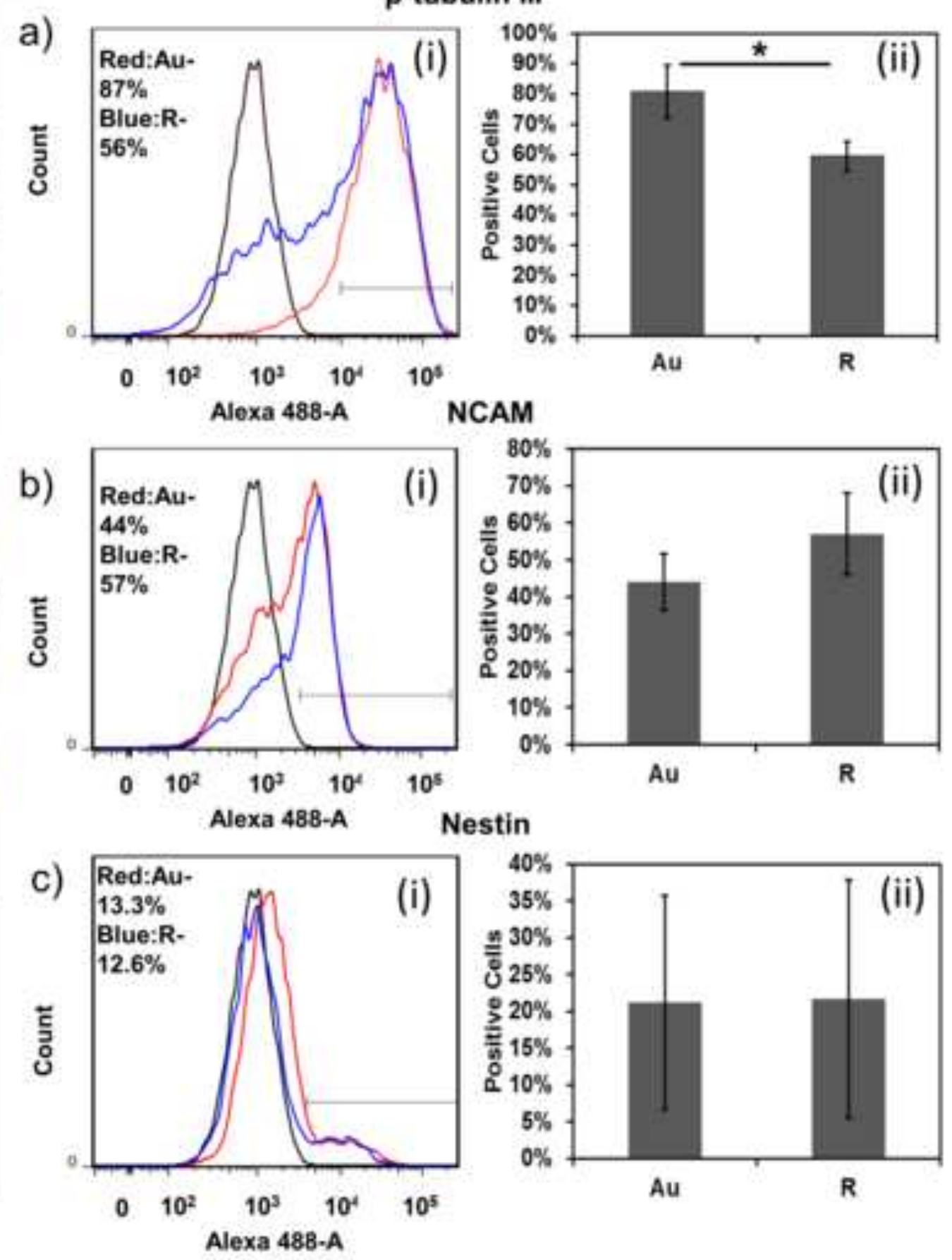
A
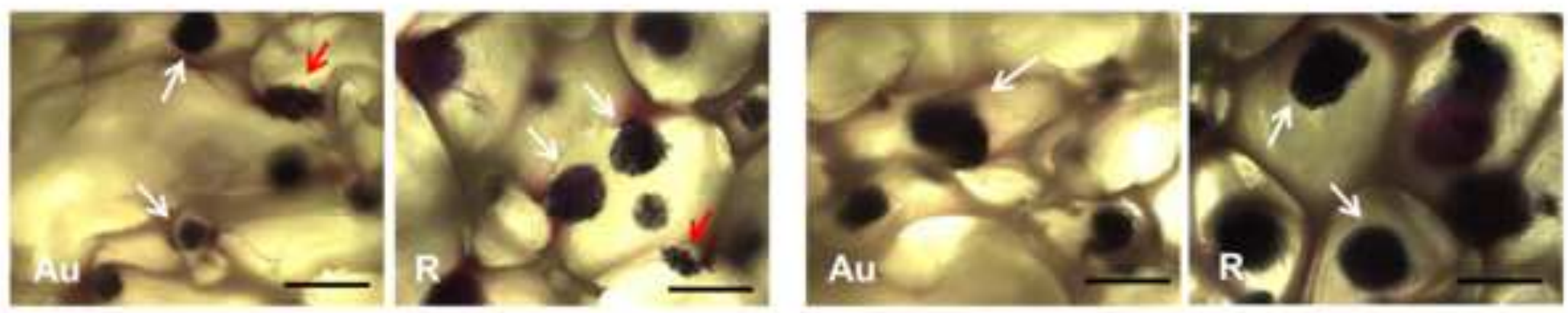

B
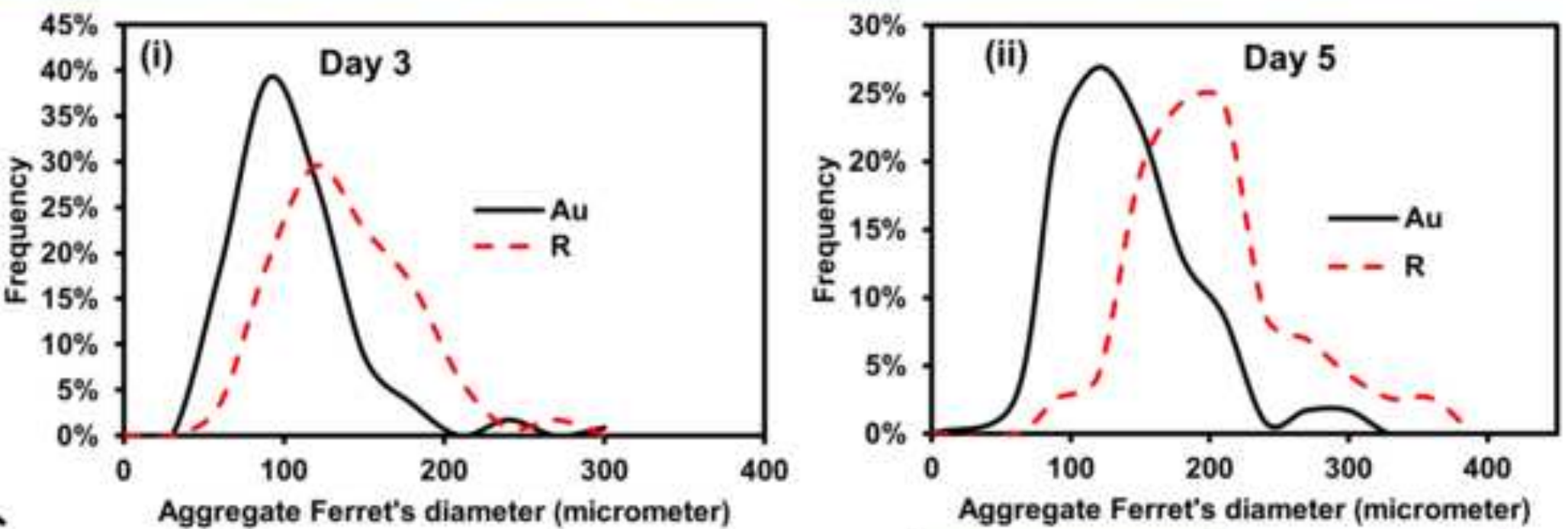

C
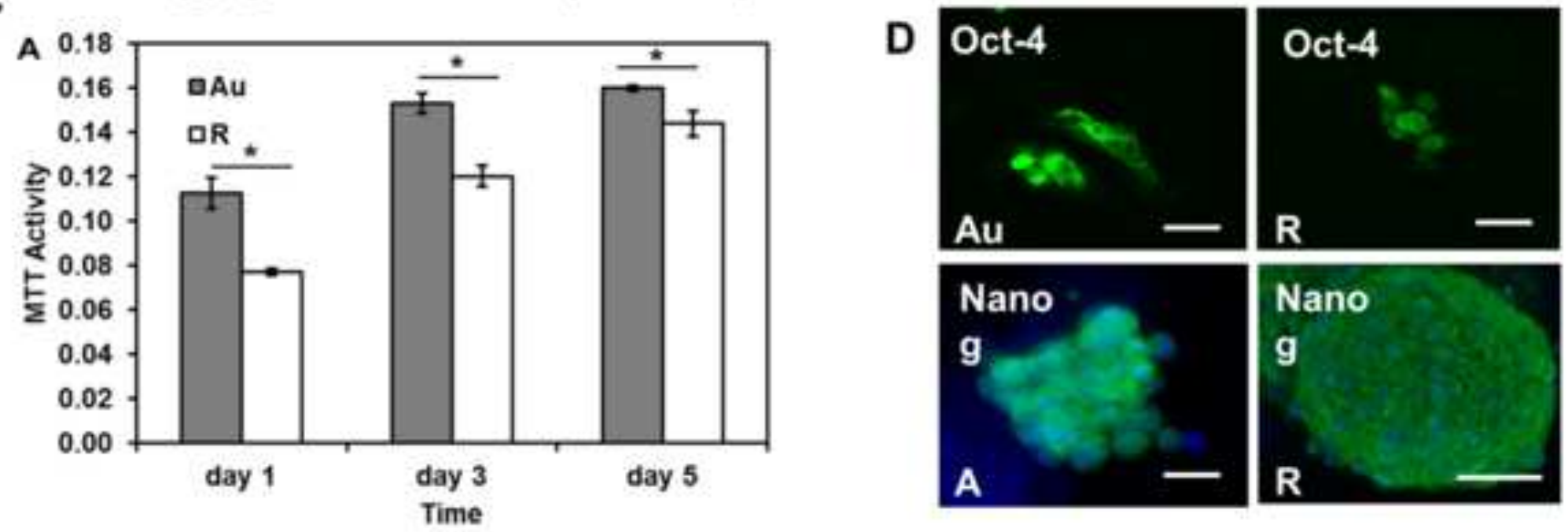

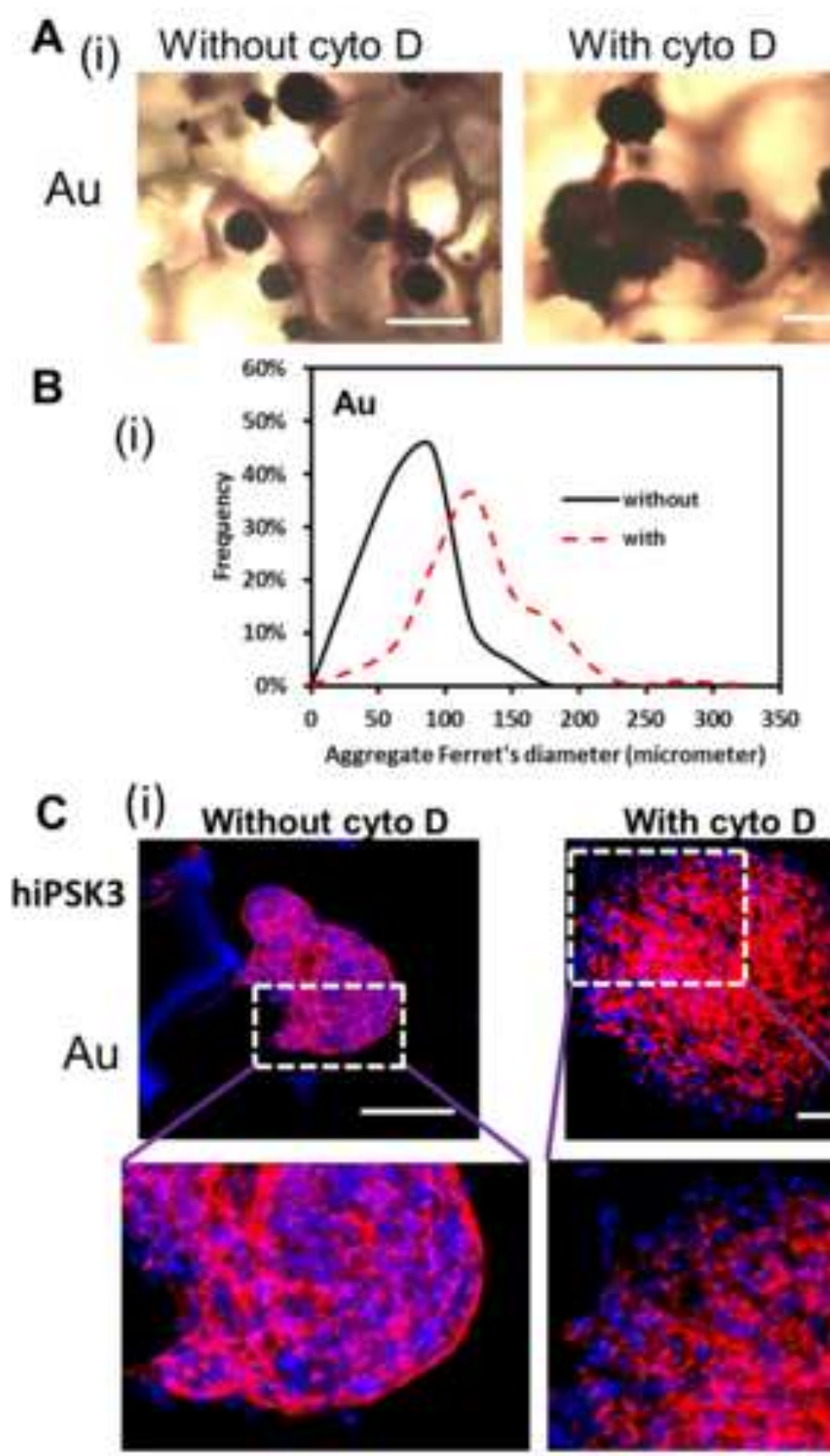

With cyto D

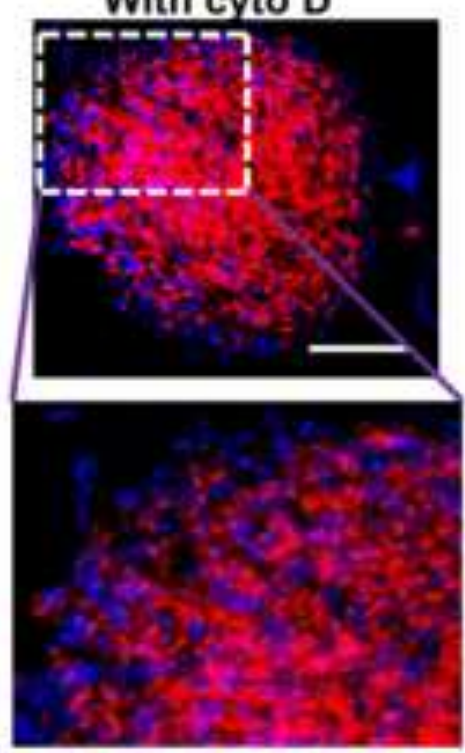

(ii) Without cyto D

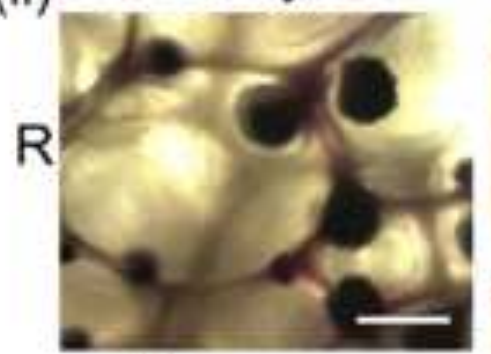

With cyto D

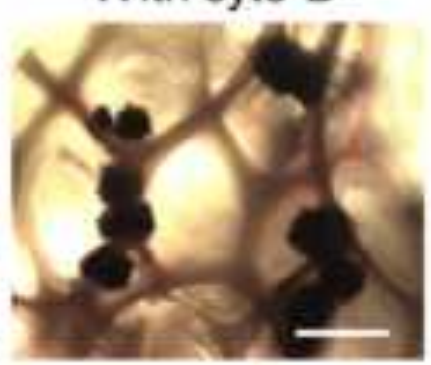

(ii)

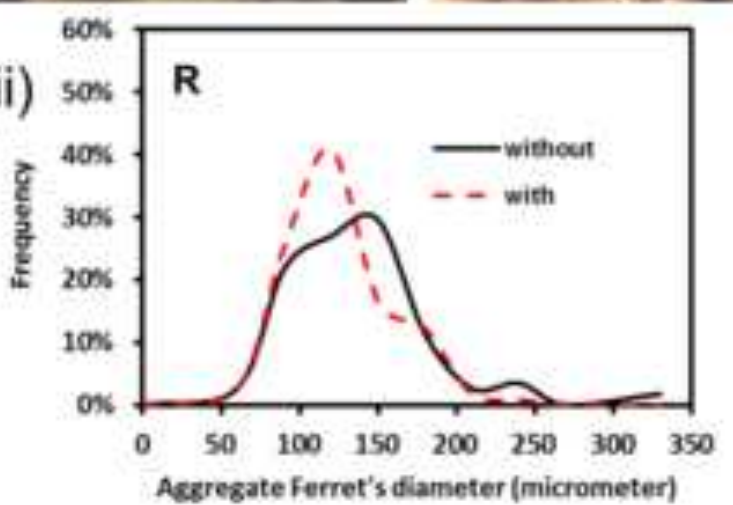

(ii)

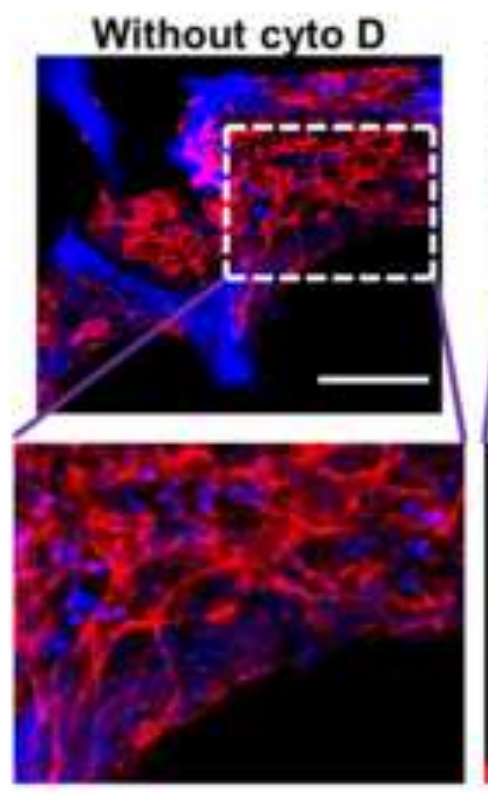

With cyto D

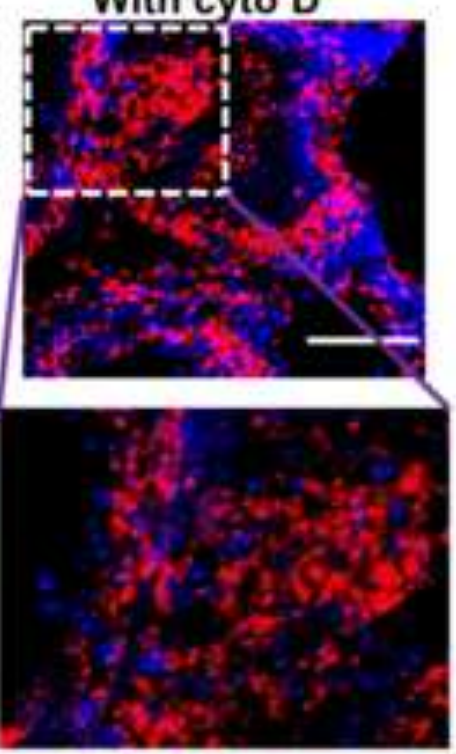



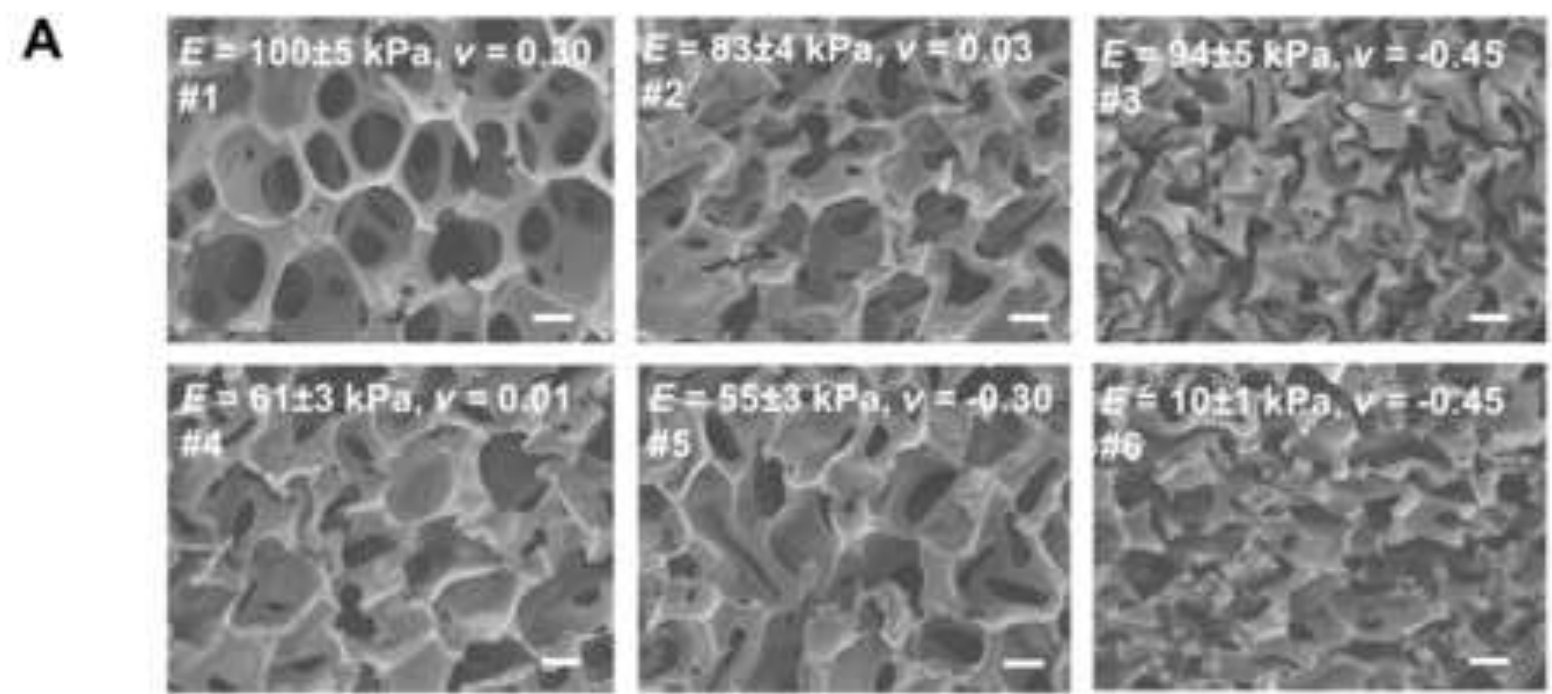

B (i)
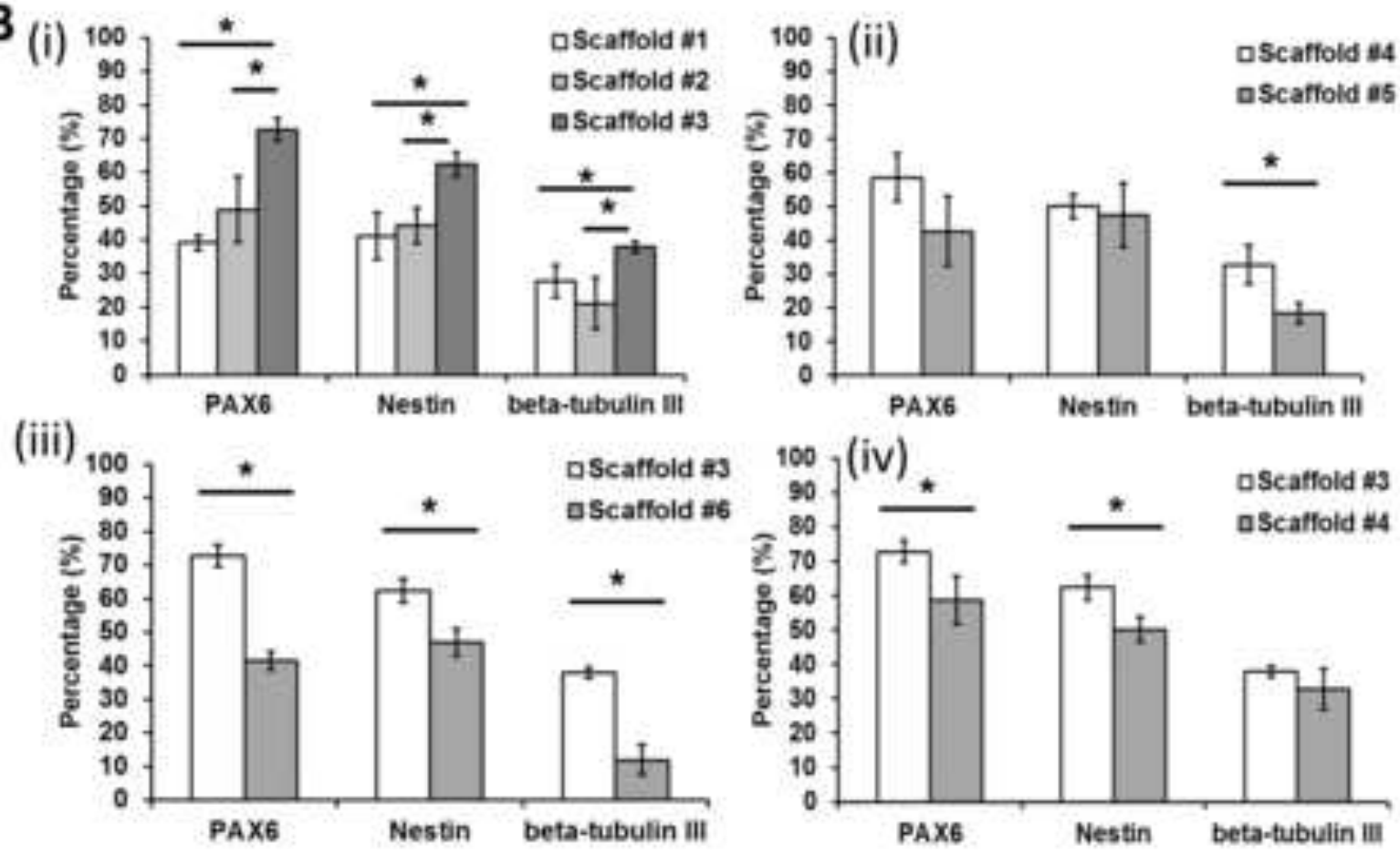


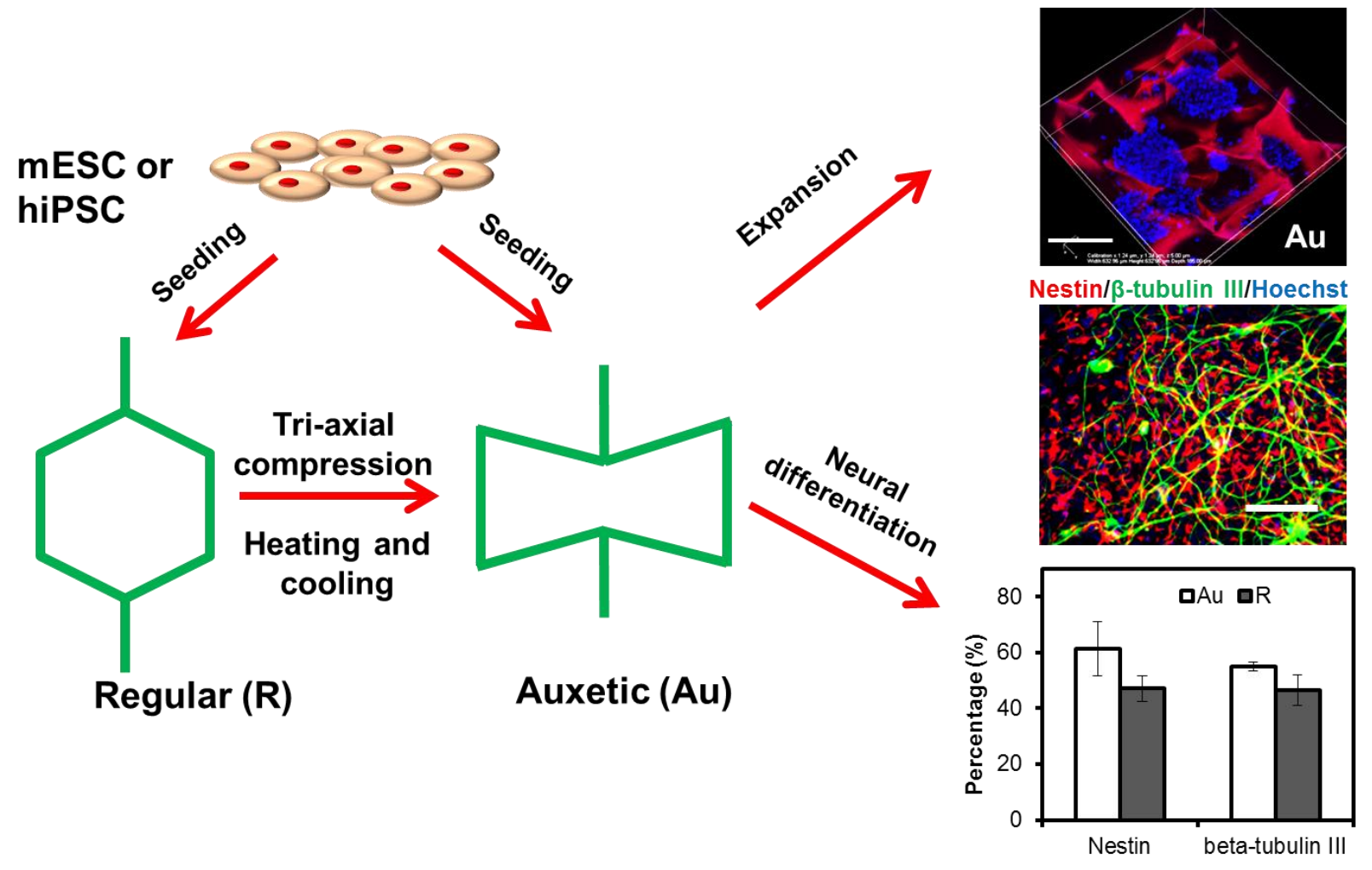

Nestin/ $\beta$-tubulin III/Hoechst 